\title{
LIFTING TO GL(2) OVER A DIVISION QUATERNION ALGEBRA, AND AN EXPLICIT CONSTRUCTION OF CAP REPRESENTATIONS
}

\author{
MASANORI MUTO, HIRO-AKI NARITA AND AMEYA PITALE
}

\begin{abstract}
The aim of this paper is to carry out an explicit construction of CAP representations of $\mathrm{GL}(2)$ over a division quaternion algebra with discriminant two. We first construct cusp forms on such a group explicitly by lifting from Maass cusp forms for the congruence subgroup $\Gamma_{0}(2)$. We show that this lifting is nonzero and Hecke-equivariant. This allows us to determine each local component of a cuspidal representation generated by such a lifting. We then show that our cuspidal representations provide examples of CAP (cuspidal representation associated to a parabolic subgroup) representations, and, in fact, counterexamples to the Ramanujan conjecture.
\end{abstract}

\section{CONTENTS}

1 Introduction 137

2 Basic notations 141

3 Some zeta integral of convolution type 147

4 Construction of the lifting 151

5 Actions of Hecke operators on the lifting 155

6 The automorphic representation corresponding to the lifting 174

References 183

\section{$\S 1$. Introduction}

One of the fundamental problems in the theory of automorphic forms or representations is to study the Ramanujan conjecture. To review it, let $\mathcal{G}$ be a reductive algebraic group over a number field $F$, and let $\mathbb{A}:=\otimes_{v \leqslant \infty}^{\prime} F_{v}$ be

Received June 21, 2014. Accepted June 11, 2015.

2010 Mathematics subject classification. 11F55, 11F70.

The second author was partly supported by Grant-in-Aid for Scientific Research (C) 24540025, Japan Society for the Promotion of Science. The third author was partly supported by National Science Foundation grant DMS-1100541.

(C) 2016 by The Editorial Board of the Nagoya Mathematical Journal 
the ring of adeles for $F$, where $F_{v}$ denotes the local field at a place $v$. The study of the Ramanujan conjecture is to consider the following question.

Question. Let $\pi=\otimes_{v \leqslant \infty}^{\prime} \pi_{v}$ be an irreducible cuspidal representation of $\mathcal{G}(\mathbb{A})$, where $\pi_{v}$ denotes the local component of $\pi$ at a place $v$. Then, is $\pi_{v}$ tempered for every $v \leqslant \infty$ ?

Nowadays it is widely known that there are cuspidal representations or cusp forms answering negatively to this question, which we call counterexamples to the Ramanujan conjecture. A well-known example is given by the SaitoKurokawa lifting to holomorphic Siegel cusp forms of degree two (cf. [17]). Another well-known example is the work by Howe and Piatetski-Shapiro [11], which gives a counterexample by a theta lifting from $\mathrm{O}(2)$ to $\mathrm{Sp}(4)$. In fact, it is expected that liftings from automorphic forms on a smaller group provide such counterexamples. On the other hand, let us recall that there is the notion of CAP representation (cuspidal representation associated to a parabolic subgroup), which was originally introduced by Piatetski-Shapiro [25]. This is a representation theoretic approach to find counterexamples to the Ramanujan conjecture.

We now note that the Ramanujan conjecture for the general linear group GL $(n)$ is strongly believed. In fact, by Jacquet and Shalika [14], it can be shown that the CAP phenomenon never occurs for $\operatorname{GL}(n)$. More generally, it is expected that the conjecture would hold for generic cuspidal representations of quasisplit reductive groups, which should be referred to as the generalized Ramanujan conjecture. In view of the Langlands functoriality principle for quasisplit groups and their inner forms, the Ramanujan conjecture for the inner forms is quite natural and interesting to study, as well as CAP representations. To define the notion of CAP representations for the inner forms we follow Gan [8] and Pitale [22] (cf. Definition 6.6). Let us note that the Saito-Kurokawa lifting deals with the case of the split symplectic group GSp(4) of degree two. The case of the nonsplit inner form $\operatorname{GSp}(1,1) \simeq \operatorname{GSpin}(1,4)$ of $\operatorname{GSp}(4)$ is considered in [8] and [22].

In this paper we take up the case of $\mathrm{GL}_{2}(B)$ over the division quaternion algebra $B$ with discriminant two. Note that $\mathrm{GL}_{2}(B)$ is an inner form of the split group GL(4). Our results provide an explicit construction of cusp forms on $\mathrm{GL}_{2}(B)$ lifted from Maass cusp forms for the congruence subgroup $\Gamma_{0}(2)$, and show that the cuspidal automorphic representations generated by such lifts are CAP representations of $\mathrm{GL}_{2}(B)$. The method of our construction of the lifting follows [22], which deals with an explicit construction of lifting 
to $\operatorname{GSp}(1,1)$. In fact, note that the cusp forms constructed by our lifting are viewed as Maass cusp forms on the 5-dimensional real hyperbolic space, while the lifting considered in [22] provides Maass cusp forms on the 4dimensional real hyperbolic space. As in [22], to prove the automorphy of our lifts, we use the converse theorem [19] by Maass, which is useful for real hyperbolic spaces of arbitrary dimension.

We explain the explicit construction of our lifting. Let $f$ be a Maass cusp form for $\Gamma_{0}(2)$ which is an eigenfunction of the Atkin-Lehner involution. Let $\{c(n)\}_{n \in \mathbb{Z} \backslash\{0\}}$ be Fourier coefficients of $f$. From the $c(n)$ s we define numbers $A(\beta)$ (cf. (4.2)) for $\beta \in B \backslash\{0\}$ in order to construct our lifting to a cusp form $F_{f}$ on $\mathrm{GL}_{2}(\mathbb{H})$ in the nonadelic setting. Actually, the $A(\beta)$ s are nothing but Fourier coefficients of $F_{f}$. The statement of our first result is as follows (cf. Theorem 4.4).

THEOREM 1.1. Let $f$ be a nonzero Mass cusp form which is an eigenfunction of the Atkin-Lehner involution. Then $F_{f}$ is a nonzero cusp form on $\mathrm{GL}_{2}(\mathbb{H})$.

Another result is that the cuspidal representations generated by the $F_{f}$ s are CAP representations of $\mathrm{GL}_{2}(B)$ and provide counterexamples to the Ramanujan conjecture. To be more precise, assume that $f$ is a Hecke eigenform. We can regard $F_{f}$ as a cusp form on the adele group $\mathcal{G}(\mathbb{A})$ with $\mathcal{G}=\mathrm{GL}_{2}(B)$. We can show that $F_{f}$ is a Hecke eigenform (cf. Section 5). Then, the strong multiplicity-one theorem proved by Badulescu and Renard [2], [3] implies that $F_{f}$ generates an irreducible cuspidal representation $\pi:=\bigotimes_{p \leqslant \infty}^{\prime} \pi_{p}$ of $\mathcal{G}(\mathbb{A})$. By our detailed study on Hecke eigenvalues of $F_{f}$, we can determine local representations $\pi_{p}$ for every $p<\infty$. We can also determine $\pi_{p}$ explicitly at $p=\infty$ by the calculation of the eigenvalue for the Casimir operator. For this we note that every $\pi_{p}$ is unramified (at $p<\infty$ ) or spherical (at $p=\infty$ ). We can show that $\pi_{p}$ (respectively $\pi_{\infty}$ ) is nontempered at every odd prime $p$ (respectively tempered at $p=\infty$ ). If we further assume that $f$ is a new form, we can also show the nontemperedness of $\pi_{p}$ at $p=2$. These lead to the following theorem (cf. Theorems 6.7, 6.8).

THEOREM 1.2.

(1) Let $f$ be a nonzero Hecke eigen cusp form, and let $F_{f}$ be the lift. Let $\sigma_{f}$ and $\pi_{F}$ be irreducible cuspidal representations generated by $f$ and $F=F_{f}$ respectively. Then $\pi_{F}$ is nearly equivalent to an irreducible constituent of $\operatorname{Ind}_{P_{2}(\mathbb{A})}^{\mathrm{GL}_{4}(\mathbb{A})}\left(|\operatorname{det}|_{\mathbb{A}}^{-1 / 2} \sigma_{f} \times|\operatorname{det}|_{\mathbb{A}}^{1 / 2} \sigma_{f}\right)$. Here, $P_{2}$ is the standard 
parabolic subgroup of $\mathrm{GL}_{4}$ with Levi subgroup $\mathrm{GL}_{2} \times \mathrm{GL}_{2}$. Namely $\pi_{F}$ is a CAP representation.

(2) The cuspidal representations $\pi_{F}$ are counterexamples to the Ramanujan conjecture.

Let $\pi^{\prime}$ be the unique irreducible quotient of $\operatorname{Ind}_{P_{2}(\mathbb{A})}^{\mathrm{GL}_{4}(\mathbb{A})}\left(|\operatorname{det}|_{\mathbb{A}}^{-1 / 2} \sigma_{f} \times\right.$ $\left.|\operatorname{det}|_{\mathbb{A}}^{1 / 2} \sigma_{f}\right)$. This is denoted by $\operatorname{MW}\left(\sigma_{f}, 2\right)$ in $[3, \S 18]$. Then $\pi^{\prime}$ is a noncuspidal, discrete series representation of $\mathrm{GL}_{4}(\mathbb{A})$. Since $\sigma_{f}$ is not the image of a cuspidal representation of $B_{\mathbb{A}}^{\times}$under the Jacquet-Langlands correspondence, $\pi^{\prime}$ is $B$-compatible according to [3, Proposition 18.2, part (a)]. Hence, there exists a discrete series representation $\pi$ of $\mathrm{GL}_{2}\left(B_{\mathbb{A}}\right)$ which maps to $\pi^{\prime}$ under the Jacquet-Langlands correspondence. Moreover, from [3, Proposition 18.2, part (b)], the representation $\pi$ has to be cuspidal. By the strong multiplicity-one theorem for $\mathrm{GL}_{2}(B)$, the representation $\pi$ has to be exactly the same as $\pi_{F}$ obtained from the classical construction. The novelty of our method is that we obtain an explicit formula for the lift in terms of Fourier expansions which are valid for non-Hecke eigenforms as well. In addition, the classical method immediately shows that the lifting is a linear nonzero map.

Let us remark that Grobner [9] has also obtained examples of CAP representations for $\mathrm{GL}_{2}(B)$ using the results of [3]. The example by Grobner [9] has a nontempered local component at the archimedean place, while our cuspidal representation $\pi$ has a tempered local component at the archimedean place, as is remarked above.

In terms of representation theory, one naturally asks whether our classical construction of the lifting is understood by a theta lifting. It is shown in [25] that the classical Saito-Kurokawa lifting is a theta lifting. In addition, [8] shows that the classical lifting to $\operatorname{GSpin}(1,4)$ obtained in [22] is also a theta lifting. We expect that the lifting constructed in this paper is also a theta lifting given by the theta correspondence between $S L(2)$ and the orthogonal group $O(5,1)$ with the Witt index $(5,1)$. One might have to use the techniques from [8] to prove such a result. We do not address this question in the current paper.

The outline of this paper is as follows. In Section 2, we first introduce the basic notation of algebraic groups and Lie groups. Next, we introduce the automorphic forms that concern us in this paper. In Section 3, we study a zeta integral attached to a Maass cusp form. This is needed in order to apply Maass converse theorem. Then, the explicit construction of 
cusp forms on $\mathrm{GL}_{2}(\mathbb{H})$ is given by lifts $F_{f}$ in Section 4. In Section 5, we view $F_{f}$ as a cusp form on the adele group $\mathcal{G}(\mathbb{A})$ and prove that, given a Hecke eigenform $f, F_{f}$ is also a Hecke eigenform at every finite place. Then, in Section 6, we determine the local components $\pi_{p}$ of the cuspidal representation $\pi$ generated by $F_{f}$ for all places $p \leqslant \infty$. We thus see that $\pi$ is a CAP representation and provides a counterexample to the Ramanujan conjecture.

\section{$\S 2 . ~ B a s i c$ notations}

\subsection{Algebraic groups, real Lie groups and the 5-dimensional hyperbolic space}

Let $B$ be the definite quaternion algebra over $\mathbb{Q}$ with discriminant $d_{B}=2$. The algebra $B$ is given by $B=\mathbb{Q}+\mathbb{Q} i+\mathbb{Q} j+\mathbb{Q} k$ with a basis $\{1, i, j, k\}$ characterized by the conditions

$$
i^{2}=j^{2}=k^{2}=-1, \quad i j=-j i=k .
$$

Let $\mathcal{G}$ be the $\mathbb{Q}$-algebraic group defined by its group of $\mathbb{Q}$-rational points

$$
\mathcal{G}(\mathbb{Q})=\mathrm{GL}_{2}(B)
$$

Here, $\mathrm{GL}_{2}(B)$ is the general linear group over $B$, which consists of elements in $M_{2}(B)$ whose reduced norms are nonzero. Let $\mathbb{H}=B \otimes_{\mathbb{Q}} \mathbb{R}$, which is nothing but the Hamilton quaternion algebra $\mathbb{R}+\mathbb{R} i+\mathbb{R} j+\mathbb{R} k$. Let $\mathbb{H} \ni x \mapsto \bar{x} \in \mathbb{H}$ denote the main involution of $\mathbb{H}$, and let $\operatorname{tr}(x)=x+\bar{x}$ and $\nu(x):=x \bar{x}$ be the reduced trace and the reduced norm of $x \in \mathbb{H}$ respectively. In what follows, we often use the notation $|\beta|:=\sqrt{\nu(\beta)}$ for $\beta \in \mathbb{H}$. We put $\mathbb{H}^{-}:=\{x \in \mathbb{H} \mid \operatorname{tr}(x)=0\}$ to be the set of pure quaternions, and $\mathbb{H}^{1}:=\{x \in$ $\mathbb{H} \mid \nu(x)=1\}$.

Denote by $G:=\mathrm{GL}_{2}(\mathbb{H})$ the general linear group of degree two with coefficients in the Hamilton quaternion algebra $\mathbb{H}$. The Lie group $G$ admits an Iwasawa decomposition

$$
G=Z^{+} N A K
$$

where

$$
Z^{+}:=\left\{\left[\begin{array}{ll}
c & 0 \\
0 & c
\end{array}\right] \mid c \in \mathbb{R}_{+}^{\times}\right\}, \quad N:=\left\{n(x)=\left[\begin{array}{ll}
1 & x \\
0 & 1
\end{array}\right] \mid x \in \mathbb{H}\right\},
$$




$$
A:=\left\{a_{y}:=\left[\begin{array}{cc}
\sqrt{y} & 0 \\
0 & \sqrt{y}^{-1}
\end{array}\right] \mid y \in \mathbb{R}_{+}^{\times}\right\}, \quad K:=\left\{\left.k \in G\right|^{t} \bar{k} k=1_{2}\right\} .
$$

The subgroup $Z^{+}$is contained in the center of $G$, and $K$ is a maximal compact subgroup of $G$, which is isomorphic to the definite symplectic group of degree two.

Let us consider the quotient $G / Z^{+} K$, which is realized as

$$
\left\{\left[\begin{array}{ll}
y & x \\
0 & 1
\end{array}\right] \mid y \in \mathbb{R}_{+}^{\times}, x \in \mathbb{H}\right\} .
$$

This gives a realization of the 5-dimensional real hyperbolic space.

\subsection{Lie algebras}

The Lie algebra $\mathfrak{g}$ of $G$ is nothing but $M_{2}(\mathbb{H})$, and has an Iwasawa decomposition

$$
\mathfrak{g}=\mathfrak{z} \oplus \mathfrak{n} \oplus \mathfrak{a} \oplus \mathfrak{k}
$$

Here,

$$
\begin{aligned}
& \mathfrak{z}:=\left\{\left[\begin{array}{ll}
c & 0 \\
0 & c
\end{array}\right] \mid c \in \mathbb{R}\right\}, \quad \mathfrak{n}:=\left\{\left[\begin{array}{ll}
0 & x \\
0 & 0
\end{array}\right] \mid x \in \mathbb{H}\right\}, \\
& \mathfrak{a}:=\left\{\left[\begin{array}{cc}
t & 0 \\
0 & -t
\end{array}\right] \mid t \in \mathbb{R}\right\}, \quad \mathfrak{k}:=\left\{X \in M_{2}(\mathbb{H}) \mid{ }^{t} \bar{X}+X=0_{2}\right\},
\end{aligned}
$$

where $\mathfrak{z}, \mathfrak{n}, \mathfrak{a}$ and $\mathfrak{k}$ are the Lie algebras of $Z^{+}, N, A$ and $K$ respectively.

We next consider the root space decomposition of $\mathfrak{g}$ with respect to $\mathfrak{a}$. Let $H:=\left[\begin{array}{cc}1 & 0 \\ 0 & -1\end{array}\right]$, and let $\alpha$ be the linear form of $\mathfrak{a}$ such that $\alpha(H)=1$. Then $\{ \pm 2 \alpha\}$ is the set of roots for $(\mathfrak{g}, \mathfrak{a})$. For $z \in \mathbb{H}$ we put

$$
E_{2 \alpha}^{(z)}:=\left[\begin{array}{cc}
0 & z \\
0 & 0
\end{array}\right], \quad E_{-2 \alpha}^{(z)}:=\left[\begin{array}{cc}
0 & 0 \\
z & 0
\end{array}\right]
$$

The set $\left\{E_{2 \alpha}^{(1)}, E_{2 \alpha}^{(i)}, E_{2 \alpha}^{(j)}, E_{2 \alpha}^{(k)}\right\}$ (respectively $\left\{E_{-2 \alpha}^{(1)}, E_{-2 \alpha}^{(i)}, E_{-2 \alpha}^{(j)}, E_{-2 \alpha}^{(k)}\right\}$ ) forms a basis of $\mathfrak{n}$ (respectively a basis of $\overline{\mathfrak{n}}:=\left\{\left[\begin{array}{ll}0 & 0 \\ x & 0\end{array}\right] \mid x \in \mathbb{H}\right\}$ ). Let $\mathfrak{z} \mathfrak{a}(\mathfrak{k}):=$ $\{X \in \mathfrak{k} \mid[X, A]=0 \forall A \in \mathfrak{a}\}$, which coincides with

$$
\left\{\left[\begin{array}{ll}
a & 0 \\
0 & d
\end{array}\right] \mid a, d \in \mathbb{H}^{-}\right\}
$$

Then $\mathfrak{z} \oplus \mathfrak{z} \mathfrak{a}(\mathfrak{k}) \oplus \mathfrak{a}$ is the eigen space with the eigenvalue zero. We then see from the root space decomposition of $\mathfrak{g}$ with respect to $\mathfrak{a}$ that $\mathfrak{g}$ decomposes 
into

$$
\mathfrak{g}=\left(\mathfrak{z} \oplus \mathfrak{z}_{\mathfrak{a}}(\mathfrak{k}) \oplus \mathfrak{a}\right) \oplus \mathfrak{n} \oplus \overline{\mathfrak{n}}
$$

We also introduce the simple Lie group $\mathrm{SL}_{2}(\mathbb{H})$ consisting of elements in $\mathrm{GL}_{2}(\mathbb{H})$ with their reduced norms 1 . The Lie algebra $\mathfrak{g}_{0}=\mathfrak{s l}_{2}(\mathbb{H})$ of $\mathrm{SL}_{2}(\mathbb{H})$ is the Lie algebra consisting of elements in $M_{2}(\mathbb{H})$ with their reduced traces zero. For this we note that

$$
\mathrm{GL}_{2}(\mathbb{H}) / Z^{+} \simeq \mathrm{SL}_{2}(\mathbb{H}), \quad \mathfrak{g} / \mathfrak{z} \simeq \mathfrak{g}_{0}
$$

We introduce the differential operator $\Omega$ defined by the infinitesimal action of

$$
\Omega:=\frac{1}{32} H^{2}-\frac{1}{4} H+\frac{1}{8} \sum_{z \in\{1, i, j, k\}} E_{2 \alpha}^{(z)^{2}} .
$$

This differential operator $\Omega$ coincides with the infinitesimal action of the Casimir element of $\mathfrak{g}_{0}$ (see [15, p. 293, Proposition 5.28]) on the space of right $K$-invariant smooth functions of $G / Z^{+}$. To check this we note that $\left[E_{2 \alpha}^{(z)}, E_{-2 \alpha}^{(\bar{z})}\right]=H$ for $z \in \mathbb{H}^{1}$ and Iwasawa decompositions $E_{-2 \alpha}^{(z)}=E_{2 \alpha}^{(\bar{z})}+$ $\left(\begin{array}{cc}0 & -\bar{z} \\ z & 0\end{array}\right)$ for $z \in \mathbb{H}$. In what follows, we call $\Omega$ the Casimir operator.

\subsection{Automorphic forms}

For $\lambda \in \mathbb{C}$ and a discrete subgroup $\Gamma \subset \mathrm{SL}_{2}(\mathbb{R})$ we denote by $S(\Gamma, \lambda)$ the space of Maass cusp forms of weight 0 on the complex upper half plane $\mathfrak{h}$ whose eigenvalue with respect to the hyperbolic Laplacian is $-\lambda$.

For a discrete subgroup $\Gamma \subset \mathrm{GL}_{2}(\mathbb{H})$ and $r \in \mathbb{C}$ we denote by $\mathcal{M}(\Gamma, r)$ the space of smooth functions $F$ on $\mathrm{GL}_{2}(\mathbb{H})$ satisfying the following conditions:

(1) $\Omega \cdot F=-\frac{1}{2}\left(r^{2} / 4+1\right) F$, where $\Omega$ is the Casimir operator defined in $(2.3)$;

(2) for any $(z, \gamma, g, k) \in Z^{+} \times \Gamma \times G \times K$, we have $F(z \gamma g k)=F(g)$;

(3) $F$ is of moderate growth.

Let $K_{\alpha}$, with $\alpha \in \mathbb{C}$, denote the modified Bessel function (see [1, s.12] $^{\text {) }}$, which satisfies the differential equation

$$
y^{2} \frac{d^{2} K_{\alpha}}{d y^{2}}+y \frac{d K_{\alpha}}{d y}-\left(y^{2}+\alpha^{2}\right) K_{\alpha}=0 .
$$


Proposition 2.1. Let $\Gamma$ be an arithmetic subgroup of $\mathrm{GL}_{2}(\mathbb{H})$, and let $L_{\Gamma}:=\{x \in \mathbb{H} \mid n(x) \in N \cap \Gamma\}$ and $\hat{L_{\Gamma}}$ be the dual lattice of $L_{\Gamma}$ with respect to tr. Then $F \in \mathcal{M}(\Gamma, r)$ admits a Fourier expansion

$$
F\left(n(x) a_{y}\right)=u(y)+\sum_{\beta \in \hat{L_{\Gamma} \backslash\{0\}}} C(\beta) y^{2} K_{\sqrt{-1} r}(4 \pi|\beta| y) e^{2 \pi \sqrt{-1} \operatorname{tr}(\beta x)},
$$

with a smooth function $u$ on $\mathbb{R}_{>0}$.

Proof. A Maass form $F \in \mathcal{M}(\Gamma ; r)$ is left-invariant with respect to $\{n(\beta) \mid$ $\left.\beta \in L_{\Gamma}\right\}$. This implies that $F(n(x+\alpha) g)=F(n(x) g)$ holds for $\alpha \in L_{\Gamma}$ and $g \in G$. Therefore, $F$ has an expansion

$$
F\left(n(x) a_{y}\right)=\sum_{\beta \in \hat{L_{\Gamma}}} W_{\beta}(y) e^{2 \pi \sqrt{-1} \operatorname{tr}(\beta x)},
$$

with a smooth function $W_{\beta}$ on $\mathbb{R}_{+}^{\times}$. For $\xi \in \mathbb{H} \backslash\{0\}$ we put $\hat{W}_{\xi}(y):=$ $y^{-3 / 2} W_{\xi}(y)$. From the condition $\Omega \cdot F=-\frac{1}{2}\left(r^{2} / 4+1\right) F$ we deduce that $\hat{W}_{\beta}$ satisfies the differential equation

$$
\left(\frac{d^{2}}{d Y^{2}}+\left(-\frac{1}{4}+\frac{\frac{1}{4}+r^{2}}{Y^{2}}\right)\right) \hat{W}_{\beta}\left(\frac{Y}{8 \pi|\beta|}\right)=0
$$

for $\beta \in \hat{L_{\Gamma}} \backslash\{0\}$, where $Y:=8 \pi|\beta| y$. This is precisely the differential equation for the Whittaker function (see $[1, \S 4.3]$ ). With the Whittaker function $W_{0, \sqrt{-1} r}$ parametrized by $(0, \sqrt{-1} r)$ we thereby see that

$$
F\left(n(x) a_{y}\right)=u(y)+\sum_{\beta \in \hat{L_{\Gamma} \backslash\{0\}}} C^{\prime}(\beta) y^{3 / 2} W_{0, \sqrt{-1} r}(8 \pi|\beta| y) e^{2 \pi \sqrt{-1} \operatorname{tr}(\beta x)},
$$

with constants $C^{\prime}(\beta)$ depending only on $\beta$. We now note the relation

$$
W_{0, \sqrt{-1} r}(2 y)=\sqrt{\frac{2 y}{\pi}} K_{\sqrt{-1} r}(y)
$$

(see [21, §13, 13.18(iii), 13.18.9]). This means that $F$ has the Fourier expansion as in the statement of the proposition.

We consider the automorphic forms above with specified discrete subgroups of $\mathrm{SL}_{2}(\mathbb{R})$ and $\mathrm{GL}_{2}(\mathbb{H})$. As a discrete subgroup of $\mathrm{SL}_{2}(\mathbb{R})$ we take the congruence subgroup $\Gamma_{0}(2)$ of level 2 . For a choice of a discrete subgroup 
of $\mathrm{GL}_{2}(\mathbb{H})$ we recall that $B$ denotes the definite quaternion algebra over $\mathbb{Q}$ with discriminant $d_{B}=2$. Up to $B^{\times}$-conjugation this has a unique maximal order. In fact, we have the maximal order $\mathcal{O}$ given by

$$
\mathcal{O}=\mathbb{Z}+\mathbb{Z} i+\mathbb{Z} j+\mathbb{Z} \frac{1+i+j+i j}{2},
$$

which is called the Hurwitz order. As a discrete subgroup of $\mathrm{GL}_{2}(\mathbb{H})$ we take $\mathrm{GL}_{2}(\mathcal{O})$.

Proposition 2.2. The group $\mathrm{GL}_{2}(\mathcal{O})$ is generated by

$$
\left\{\left[\begin{array}{cc}
0 & 1 \\
-1 & 0
\end{array}\right],\left[\begin{array}{ll}
u & 0 \\
0 & 1
\end{array}\right],\left[\begin{array}{ll}
1 & v \\
0 & 1
\end{array}\right] \mid u \in \mathcal{O}^{\times}, v \in \mathcal{O}\right\} .
$$

Proof. Any element of the form $\left[\begin{array}{ll}\alpha & \beta \\ 0 & \delta\end{array}\right] \in \mathrm{GL}_{2}(\mathcal{O})$ can be expressed as

$$
\left[\begin{array}{ll}
\alpha & 0 \\
0 & 1
\end{array}\right]\left[\begin{array}{ll}
1 & 0 \\
0 & \delta
\end{array}\right]\left[\begin{array}{cc}
1 & \alpha^{-1} \beta \\
0 & 1
\end{array}\right]
$$

We see that $\alpha, \delta \in \mathcal{O}^{\times}$, and thus $\alpha^{-1} \beta \in \mathcal{O}$. We note that

$$
\left[\begin{array}{cc}
0 & 1 \\
-1 & 0
\end{array}\right]\left[\begin{array}{ll}
u & 0 \\
0 & 1
\end{array}\right]\left[\begin{array}{cc}
0 & -1 \\
1 & 0
\end{array}\right]=\left[\begin{array}{ll}
1 & 0 \\
0 & u
\end{array}\right], \quad\left[\begin{array}{cc}
0 & 1 \\
-1 & 0
\end{array}\right]^{3}=\left[\begin{array}{cc}
0 & -1 \\
1 & 0
\end{array}\right] .
$$

These imply the assertion for $\left[\begin{array}{ll}\alpha & \beta \\ 0 & \delta\end{array}\right] \in \mathrm{GL}_{2}(\mathcal{O})$. Next, we have the following claim.

For $a, b \in \mathcal{O}$ with $b \neq 0$ there exist $c, d \in \mathcal{O}$ such that $a=c b+d, \quad \nu(d)<$ $\nu(b)$. This follows from [16, Chap. I, Section 1, Corollary 1.8]. This reduces the general case of $\left[\begin{array}{ll}\alpha & \beta \\ \gamma & \delta\end{array}\right] \in \mathrm{GL}_{2}(\mathcal{O}), \gamma \neq 0$, to the previous case. This completes the proof of the proposition.

Let $\mathcal{G}(\mathbb{A})=\mathrm{GL}_{2}\left(B_{\mathbb{A}}\right)$, where $B_{\mathbb{A}}$ denotes the adelization of $B$, and let $U$ be the compact subgroup of $\mathcal{G}(\mathbb{A})$ given by $\prod_{p<\infty} \mathrm{GL}_{2}\left(\mathcal{O}_{p}\right)$, where $\mathcal{O}_{p}$ denotes the $p$-adic completion of $\mathcal{O}$ at a finite prime $p$. Then, the class number of $\mathcal{G}$ with respect to $U$ is defined as the number of cosets in $U \mathcal{G}(\mathbb{R}) \backslash \mathcal{G}(\mathbb{A}) / \mathcal{G}(\mathbb{Q})$.

We next put $\mathcal{P}$ to be a standard $\mathbb{Q}$-parabolic subgroup of $\mathcal{G}$ whose group of $\mathbb{Q}$-rational points is $\mathcal{P}(\mathbb{Q})=\left\{\left[\begin{array}{ll}\alpha & \beta \\ 0 & \delta\end{array}\right] \in \mathcal{G}(\mathbb{Q})\right\}$. We now recall that, for an arithmetic subgroup $\Gamma \subset \mathcal{G}(\mathbb{Q})$, the cosets $\Gamma \backslash \mathcal{G}(\mathbb{Q}) / \mathcal{P}(\mathbb{Q})$ are called the set of $\Gamma$-cusps. 
LEMMA 2.3.

(1) The class number of $\mathcal{G}$ with respect to $U$ is one, namely we have $\mathcal{G}(\mathbb{A})=$ $\mathcal{G}(\mathbb{Q}) \mathcal{G}(\mathbb{R}) U$.

(2) The number of cusps with respect to $\Gamma:=\mathrm{GL}_{2}(\mathcal{O})$ is one.

Proof. According to [24, Theorem 8.11] the class number of a reductive group over a number field $F$ is not greater than that of a parabolic $F$ subgroup of it. Furthermore, the class number of a parabolic $F$-subgroup is not greater than that of its Levi subgroup (see [24, Proposition 5.4]). Hence, the class number of $\mathcal{G}$ with respect to $U$ turns out to be not greater than that of the Levi subgroup $\mathcal{L}$ defined by the $\mathbb{Q}$-rational points $B^{\times} \times B^{\times}$. Since the class number of $B^{\times}$with respect to $\prod_{p<\infty} \mathcal{O}_{p}^{\times}$is one, the class number of $\mathcal{G}$ is also one, which means that $\mathcal{G}(\mathbb{A})=\mathcal{G}(\mathbb{Q}) \mathcal{G}(\mathbb{R}) U=U \mathcal{G}(\mathbb{R}) \mathcal{G}(\mathbb{Q})$. This completes the proof of (1).

The first assertion implies that there is a bijection

$$
\Gamma \backslash \mathcal{G}(\mathbb{Q}) / \mathcal{P}(\mathbb{Q}) \simeq \mathcal{G}(\mathbb{R}) U \backslash \mathcal{G}(\mathbb{A}) / \mathcal{P}(\mathbb{Q}) .
$$

Furthermore, note an Iwasawa decomposition

$$
\mathcal{G}\left(\mathbb{Q}_{v}\right)= \begin{cases}\mathrm{GL}_{2}\left(\mathcal{O}_{p}\right) \cdot P\left(\mathbb{Q}_{p}\right) & (v=p<\infty) \\ K \cdot P(\mathbb{R}) & (v=\infty)\end{cases}
$$

at every place $v \leqslant \infty$. We can then reduce the counting of the number of $\Gamma$-cusps to that of the class number of the Levi subgroup $\mathcal{L}$. This implies that the number of cusps with respect to $\Gamma$ is one, and completes the proof of the lemma.

We define $\Gamma_{T}$ as a subgroup $\mathrm{GL}_{2}(\mathcal{O})$ generated by

$$
\left[\begin{array}{cc}
0 & -1 \\
1 & 0
\end{array}\right], \quad\left[\begin{array}{ll}
1 & \beta \\
0 & 1
\end{array}\right] \quad(\beta \in \mathcal{O}) .
$$

In what follows, we deal mainly with $S\left(\Gamma_{0}(2) ;-\left(\frac{1}{4}+\left(\frac{r}{2}\right)^{2}\right)\right), \mathcal{M}\left(\Gamma_{T} ; r\right)$ and $\mathcal{M}\left(\mathrm{GL}_{2}(\mathcal{O}), r\right)$. For this we should note that the Selberg conjecture for $\Gamma_{0}(2)$ is verified (cf. [12, Corollary 11.5]). This means the following.

Proposition 2.4. If $S\left(\Gamma_{0}(2) ;-\left(\frac{1}{4}+(r / 2)^{2}\right)\right) \neq\{0\}$, we have $\frac{1}{4}+$ $(r / 2)^{2} \geqslant \frac{1}{4}$, namely we can assume that $r \in \mathbb{R}$. 
By Proposition 2.1, the Fourier expansion of $F \in \mathcal{M}\left(\mathrm{GL}_{2}(\mathcal{O}), r\right)$ is then written as

$$
\begin{aligned}
F\left(n(x) a_{y}\right) & =u(y)+\sum_{\beta \in \frac{1}{2} S \backslash\{0\}} C(\beta) y^{2} K_{\sqrt{-1} r}(4 \pi \nu(\beta) y) e^{2 \pi \sqrt{-1} \operatorname{tr}(\beta x)} \\
& =u(y)+\sum_{\beta \in S \backslash\{0\}} A(\beta) y^{2} K_{\sqrt{-1} r}(2 \pi|\beta| y) e^{2 \pi \sqrt{-1} \operatorname{Re}(\beta x)},
\end{aligned}
$$

with a smooth function $u$ on $\mathbb{R}_{>0}$. Here,

$$
S:=\mathbb{Z} \cdot(1-i j)+\mathbb{Z} \cdot(-i-i j)+\mathbb{Z} \cdot(-j-i j)+\mathbb{Z} \cdot 2 i j
$$

is the dual lattice of $\mathcal{O}$ with respect to the bilinear form on $\mathbb{H} \times \mathbb{H}$ defined by $\operatorname{Re}=\frac{1}{2}$ tr.

Now we introduce $\varpi_{2}=1+i$, which is a uniformizer of $B \otimes \mathbb{Q}_{2}$. We can verify the following lemma by a direct computation.

LEMMA 2.5. We have $S=\varpi_{2} \mathcal{O}$.

\section{$\S 3$. Some zeta integral of convolution type}

In this section, we study certain zeta integrals which play a crucial role in the proof of automorphy in Section 4.

\section{Theta functions}

Consider the space of harmonic polynomials of degree $l$ on $\mathbb{H}$. These are homogeneous polynomials of degree $l$ and are annihilated by the Laplace operator in 4 variables. We can act on this space by the cyclic group of order 8 generated by $\frac{1+i}{\sqrt{2}} \in \mathbb{H}$. Let $\left\{P_{l, \nu}\right\}_{\nu}$ denote a basis for this space consisting of eigenvectors under the above action. Hence,

$$
P_{l, \nu}\left(\frac{1+i}{\sqrt{2}} x\right)=\epsilon_{l, \nu} P_{l, \nu}(x),
$$

for some 8 th root of unity $\epsilon_{l, v}$. Define the following theta function:

$$
\Theta_{l, \nu}(z):=\sum_{\beta \in S} P_{l, \nu}(\beta) e^{2 \pi \sqrt{-1} \frac{|\beta|^{2}}{2} z}=\sum_{m=0}^{\infty} b(2 m) e^{2 \pi \sqrt{-1} m z}
$$

on the complex upper half plane $\mathfrak{h}$, where $b(m):=\sum_{\beta \in S,|\beta|^{2}=m} P_{l, \nu}(\beta)$. Since $S$ is invariant under $\beta \mapsto-\beta$ and $P_{l, \nu}(-x)=(-1)^{l} P_{l, \nu}(x)$, we see that $\Theta_{l, \nu}(z)$ is the zero function if $l$ is odd.

LEMMA 3.1. Let $l$ be an even nonnegative integer. Let $\Theta_{l, \nu}$ be as defined in (3.2), with $P_{l, \nu}$ satisfying (3.1). Then $\Theta_{l, \nu}$ is a holomorphic modular form 
of weight $l+2$ with respect to $\Gamma_{0}(2)$, and is a cusp form if $l \geqslant 2$. Moreover, we have the following transformation formula:

$$
\Theta_{l, \nu}\left(\frac{-1}{2 z}\right)=-\epsilon_{l, \nu}^{-1} 2^{l / 2+1} z^{l+2} \Theta_{l, \nu}(z) .
$$

Proof. Set

$$
B=\left[\begin{array}{cccc}
1 & & & \\
& 1 & & \\
-1 & -1 & -1 & 2
\end{array}\right] \text { and } A={ }^{t} B B
$$

By (2.5), we see that the map $x \mapsto B x$ is a bijection from $\mathbb{Z}^{4} \simeq \mathbb{Z}+\mathbb{Z} i+$ $\mathbb{Z} j+\mathbb{Z} k$ to $B\left(\mathbb{Z}^{4}\right) \simeq S$. Here, we consider $x$ as a column vector. For $P_{l, \nu}$ in the statement of the lemma, set $P(x):=P_{l, \nu}(B x), x \in \mathbb{Z}^{4}$. Then $P$ is a homogeneous polynomial in 4 variables of degree $l$ annihilated by the operator

$$
\Delta_{A}=\sum_{i, j=1}^{4} b_{i, j} \frac{\partial^{2}}{\partial x_{i} \partial x_{j}}, \quad \text { where } A^{-1}=\left(b_{i, j}\right) .
$$

One can then see that $\Theta_{l, \nu}(z)=\Theta(z ; A, P)$, where

$$
\Theta(z ; A, P)=\sum_{m \in \mathbb{Z}^{4}} P(m) e^{2 \pi \sqrt{-1} \frac{t^{t} m A m}{2} z}
$$

is as defined in [20, Corollary 4.9.5]. Since all diagonal entries of $A$ and $2 A^{-1}$ are even, [20, part (3) of Corollary 4.9.5] implies that $\Theta_{l, \nu}$ is a holomorphic modular form of weight $l+2$ with respect to $\Gamma_{0}(2)$, and is a cusp form if $l \geqslant 2$. Once again, by [20, part (3) of Corollary 4.9.5], we have

$$
\Theta\left(\frac{-1}{2 z} ; A, P\right)=-2^{l+1} z^{l+2} \Theta\left(z ; A^{*}, P^{*}\right),
$$

where $A^{*}=2 A^{-1}$ and $P^{*}(x)=P\left(A^{-1} x\right)=P_{l, \nu}\left({ }^{t} B^{-1} x\right)$. Note that the map $x \mapsto{ }^{t} B^{-1} x$ can be regarded as a bijection between $\mathbb{Z}^{4}$ and $\mathcal{O}$. Hence, we see that

$$
\begin{aligned}
\Theta\left(z ; A^{*}, P^{*}\right) & =\sum_{\beta \in \mathcal{O}} P_{l, \nu}(\beta) e^{2 \pi \sqrt{-1}|\beta|^{2} z} \\
& =\epsilon_{l, \nu}^{-1} 2^{-l / 2} \sum_{m=0}^{\infty} b(2 m) e^{2 \pi \sqrt{-1} m z}=\epsilon_{l, \nu}^{-1} 2^{-l / 2} \Theta_{l, \nu}(z) .
\end{aligned}
$$


Here, we have used Lemma 2.5 and (3.1). This completes the proof of the lemma.

Eisenstein series with respect to $\Gamma_{0}(2)$

We introduce an Eisenstein series

$$
\begin{aligned}
\tilde{E}_{\infty}(z, s):= & (4 \pi)^{l / 2} \frac{\Gamma\left(s+\frac{l}{2}+1\right)}{\Gamma(s)}\left(\pi^{-s} \Gamma(s) \zeta(2 s)\right) \frac{1}{2} \\
& \times \sum_{\gamma \in \Gamma_{\infty} \backslash \Gamma_{0}(2)}\left(\frac{c z+d}{|c z+d|}\right)^{l+2}\left(\frac{\operatorname{Im}(z)}{|c z+d|^{2}}\right)^{s}
\end{aligned}
$$

on $\mathfrak{h}$ with a complex parameter $s$, where $\Gamma_{\infty}:=\left\{\left[\begin{array}{cc}1 & m \\ 0 & 1\end{array}\right] \mid m \in \mathbb{Z}\right\}$. The Eisenstein series satisfies the following functional equation.

Lemma 3.2. Let $\tilde{E}_{0}(z, s):=(z /|z|)^{l+2} \tilde{E}_{\infty}(-1 / 2 z, s)$. Then the functional equation

$$
\tilde{E}_{\infty}(z, 1-s)=\frac{2^{2 s-2}}{1-2^{2 s-2}} \tilde{E}_{\infty}(z, s)+\frac{2^{s-1}\left(1-2^{2 s-1}\right)}{1-2^{2 s-2}} \tilde{E}_{0}(z, s)
$$

holds.

Proof. This is settled by the argument in [7, Lemma 7] with the help of the formula for the scattering matrices in $[12, \S 11.2]$.

Let $f \in S\left(\Gamma_{0}(2) ;-\left(\frac{1}{4}+(r / 2)^{2}\right)\right)$ be an eigenfunction with respect to the Atkin-Lehner involution; that is, $f(-1 / 2 z)=\epsilon f(z)$ with some $\epsilon \in\{ \pm 1\}$. Suppose the form $f$ has the Fourier expansion

$$
f(x+\sqrt{-1} y)=\sum_{n \in \mathbb{Z} \backslash\{0\}} c(n) W_{0, \frac{\sqrt{-1} r}{2}}(4 \pi|n| y) e^{2 \pi \sqrt{-1} n x},
$$

where $W_{0, \sqrt{-1} r / 2}$ denotes the Whittaker function with the parameter $(0, \sqrt{-1} r / 2)$. Let $l$ be an even nonnegative integer. Let $\Theta_{l, \nu}$ be as defined in (3.2), with $P_{l, \nu}$ satisfying (3.1). Let $\tilde{E}_{\infty}(z, s)$ be the Eisenstein series defined in (3.4). Let the zeta integral $I(s)$ be defined by

$$
I(s):=\int_{\Gamma_{0}(2) \backslash \mathfrak{h}} f(z) \Theta_{l, \nu}(z) \tilde{E}_{\infty}(z, s) y^{l+2 / 2} \frac{d x d y}{y^{2}} .
$$

A similar Rankin-Selberg integral was considered in [7, Theorem 6] (see also $[22,(3.16)])$. This integral is the main tool to prove the analytic properties of certain Dirichlet series associated with the lifting defined in Section 4. 
We would like to remark that the integral $I(s)$ is, in general, not Eulerian and does not represent an $L$-function of $f$.

By Lemma 3.1, the above integral is well defined. Let us now state the theorem of this section.

THEOREM 3.3. The zeta integral $I(s)$ is entire and is bounded on vertical strips. When $\epsilon \epsilon_{l, \nu}=1$, we have

$$
\left(2^{s}-1\right) I(s)=\left(2^{1-s}-1\right) I(1-s) .
$$

When $\epsilon \epsilon_{l, \nu}=-1$, we have

$$
\left(2^{s}+1\right) I(s)=\left(2^{1-s}+1\right) I(1-s) .
$$

Proof. The entireness and boundedness on vertical strips of $I(s)$ are verified by the same argument as $[22, \S 3.2]$. We put

$$
I_{0}(s):=\int_{\Gamma_{0}(2) \backslash \mathfrak{h}} f(z) \Theta_{l, \nu}(z) \tilde{E}_{0}(z, s) y^{l+2 / 2} \frac{d x d y}{y^{2}} .
$$

Since $\Gamma_{0}(2)$ is stable under conjugation by $[-\sqrt{2} 1 / \sqrt{2}] \in \mathrm{SL}_{2}(\mathbb{R})$, we can make a change of variable $z \mapsto-1 /(2 z)$. Now, using the assumption $f(-1 / 2 z)=\epsilon f(z),(3.3)$ and the definition of $\tilde{E}_{0}(z, s)$, we have

$$
\begin{aligned}
I_{0}(s)= & \int_{\Gamma_{0}(2) \backslash \mathfrak{h}} f\left(-\frac{1}{2 z}\right) \Theta_{l, \nu}\left(-\frac{1}{2 z}\right) \tilde{E}_{0}\left(-\frac{1}{2 z}, s\right) \operatorname{Im}\left(\frac{-1}{2 z}\right)^{l+2 / 2} \frac{d x d y}{y^{2}} \\
= & \int_{\Gamma_{0}(2) \backslash \mathfrak{h}}[\epsilon f(z)]\left[-\epsilon_{l, \nu}^{-1} 2^{l / 2+1} z^{l+2} \Theta_{l, \nu}(z)\right] \\
& \times\left[\left(\frac{-|z|}{z}\right)^{l+2} \tilde{E}_{\infty}(z, s)\right]\left[\frac{y}{2|z|^{2}}\right]^{l+2 / 2} \frac{d x d y}{y^{2}} \\
= & -\epsilon_{l, v}^{-1} \in I(s) .
\end{aligned}
$$

From Lemma 3.2, we deduce

$$
I(1-s)=\frac{2^{2 s-2}}{1-2^{2 s-2}} I(s)+\frac{2^{s-1}\left(1-2^{2 s-1}\right)}{1-2^{2 s-2}} I_{0}(s) .
$$

Observe that

$$
\frac{2^{2 s-2}}{1-2^{2 s-2}}-\epsilon_{l, \nu}^{-1} \epsilon \frac{2^{s-1}\left(1-2^{2 s-1}\right)}{1-2^{2 s-2}}= \begin{cases}\frac{2^{s}-1}{2^{1-s}-1} & \text { if } \epsilon_{l, \nu} \epsilon=1 \\ \frac{2^{s}+1}{2^{1-s}+1} & \text { if } \epsilon_{l, \nu} \epsilon=-1 .\end{cases}
$$

We have therefore proved the theorem. 


\section{$\S 4$. Construction of the lifting}

We now construct a lifting map from $S\left(\Gamma_{0}(2) ;-\left(\frac{1}{4}+r^{2} / 4\right)\right)$ to $\mathcal{M}\left(\mathrm{GL}_{2}(\mathcal{O}) ; r\right)$, which is an analog of that of Pitale [22]. The fundamental tool of our study is the converse theorem by Maass [19].

Theorem 4.1. (Maass) Let $\{A(\beta)\}_{\beta \in S \backslash\{0\}}$ be a sequence of complex numbers such that

$$
A(\beta)=O\left(|\beta|^{\kappa}\right) \quad(\exists \kappa>0),
$$

and put

$$
F\left(n(x) a_{y}\right):=\sum_{\beta \in S \backslash\{0\}} A(\beta) y^{2} K_{\sqrt{-1} r}(2 \pi|\beta| y) e^{2 \pi \sqrt{-1} \operatorname{Re}(\beta x)} .
$$

For a harmonic polynomial $P$ on $\mathbb{H}$ of degree l we introduce

$$
\xi(s, P):=\pi^{-2 s} \Gamma\left(s+\frac{\sqrt{-1} r}{2}\right) \Gamma\left(s-\frac{\sqrt{-1} r}{2}\right) \sum_{\beta \in S \backslash\{0\}} A(\beta) \frac{P(\beta)}{|\beta|^{2 s}},
$$

which converges for $\operatorname{Re}(s)>l+4+\kappa / 2$. Let $\left\{P_{l, \nu}\right\}_{\nu}$ be a basis of harmonic polynomials on $\mathbb{H}$ of degree $l$.

Then $F \in \mathcal{M}\left(\Gamma_{T} ; r\right)$ is equivalent to the condition that, for any $l, \nu$, the $\xi\left(s, P_{l, \nu}\right)$ satisfies the following three conditions.

(1) It has analytic continuation to the whole complex plane.

(2) It is bounded on any vertical strip of the complex plane.

(3) The functional equation

$$
\xi\left(2+l-s, P_{l, \nu}\right)=(-1)^{l} \xi\left(s, \hat{P}_{l, \nu}\right)
$$

holds, where $\hat{P}(x):=P(\bar{x})$ for $x \in \mathbb{H}$.

Recall that $\Gamma_{T}$ is defined in (2.4). For this theorem we remark that the infinitesimal action of the Casimir operator $\Omega$ on the space of smooth right $K$-invariant functions on $G / Z$ can be identified with a constant multiple of the hyperbolic Laplacian on $\left\{\left[\begin{array}{ll}y & x \\ 0 & 1\end{array}\right] \mid x \in \mathbb{H}, y \in \mathbb{R}_{+}^{\times}\right\}$(for the hyperbolic Laplacian, see $[19,(3)])$. We can therefore follow the argument in $[19, \S 2]$ to see that this theorem is useful also for our situation.

We wish to define $\{A(\beta)\}_{\beta \in S \backslash\{0\}}$ from Fourier coefficients $c(n)$ of $f \in S\left(\Gamma_{0}(2) ;-\left(\frac{1}{4}+r^{2} / 4\right)\right)$. Let $\varpi_{2}=1+i$, as before. An easy computation shows that $\varpi_{2} \mathcal{O}=\mathcal{O} \varpi_{2}$. This allows us to write any $\beta \in \mathcal{O}$ uniquely as 
$\beta=\varpi_{2}^{u} d \beta^{\prime}$, where $u \geqslant 0, d$ is an odd integer and $\beta^{\prime} \in \mathcal{O}$ is neither of the form $\varpi_{2} \beta_{0}^{\prime}$ with some nonzero $\beta_{0}^{\prime} \in \mathcal{O}$ nor a multiple of an element of $\mathcal{O}$ by an odd integer. Hence, we can define $\varpi_{2}^{m} \mid \beta$ by $m \leqslant u$ with $u$ as above. Recall that, by Lemma 2.5, we have $S=\varpi_{2} \mathcal{O}$. It thus makes sense to define the set $S^{\text {prim }}$ of primitive elements in $S$ by

$$
S^{\text {prim }}:=\left\{\beta \in S \backslash\{0\}\left|\varpi_{2}\right| \beta, \varpi_{2}^{2} \nmid \beta, d \nmid \beta \text { for all odd integers } d\right\},
$$

where $d \nmid \beta$ for $d \in \mathbb{Z}$ means that $\beta$ is not a multiple of an element in $S$ by $d$.

Proposition 4.2. Let $\beta \in S \backslash\{0\}$ be expressed as

$$
\beta=\varpi_{2}^{u} d \beta_{0}
$$

where $u$ is a nonnegative integer, $d$ is an odd integer and $\beta_{0} \in S^{\text {prim }}$. Given $f \in S\left(\Gamma_{0}(2) ;-\left(\frac{1}{4}+r^{2} / 4\right)\right)$ with Fourier coefficients $c(n)$ and eigenvalue $\epsilon \in$ $\{ \pm 1\}$ of the Atkin-Lehner involution, we set

$$
A(\beta):=|\beta| \sum_{t=0}^{u} \sum_{n \mid d}(-\epsilon)^{t} c\left(-\frac{|\beta|^{2}}{2^{t+1} n^{2}}\right) .
$$

Let $\left\{P_{l, \nu}\right\}_{\nu}$ be a basis of harmonic polynomials on $\mathbb{H}$ of degree $l$ satisfying (3.1). Then we have

$$
\xi\left(s+\frac{l}{2}+\frac{1}{2}, P_{l, \nu}\right)= \begin{cases}2^{1-l / 2} \pi^{-(l+1)}\left(2^{s}-\epsilon \epsilon_{l, \nu}\right) I(s) & \text { if } \epsilon_{l, \nu} \in\{ \pm 1\} \\ 0 & \text { if } \epsilon_{l, \nu} \notin\{ \pm 1\}\end{cases}
$$

and the $\xi\left(s, P_{l, \nu}\right)$ satisfies the three analytic conditions in Theorem 4.1.

Proof. Note that, if $l$ is odd, then $\xi\left(s, P_{l, \nu}\right) \equiv 0$, since $A(\beta)$ is invariant under $\beta \mapsto-\beta$ and $P_{l, \nu}$ is homogeneous of degree $l$. For this we remark that, from Section $3, I(s) \equiv 0$ also holds when $l$ is odd. From now on we assume that $l$ is even. Now suppose that the condition $\epsilon_{l, \nu} \notin\{ \pm 1\}$ is satisfied, which is equivalent to $\epsilon_{l, \nu}^{2} \neq 1$. We see that $P_{l, \nu}(i \beta)=\epsilon_{l, \nu}^{2} P_{l, \nu}(\beta) \neq P_{l, \nu}(\beta)$ for $\beta \in \mathbb{H}$. In addition, we note that $A(i \beta)|i \beta|^{-2 s}=A(\beta)|\beta|^{-2 s}$ for $\beta \in S$. In the definition of $\xi\left(s, P_{l, \nu}\right)$, we can replace $S$ by $i S$. Hence, we obtain $\xi\left(s, P_{l, \nu}\right) \equiv 0$, if $\epsilon_{l, \nu} \notin\{ \pm 1\}$.

We now assume that $\epsilon_{l, \nu} \in\{ \pm 1\}$. By a formal calculation similar to [22, Proposition 3.5] we get 


$$
\begin{aligned}
I(s)= & \pi^{-2 s} 2^{-2 s-1} \Gamma\left(s+\frac{l}{2}+\frac{1}{2}+\frac{\sqrt{-1} r}{2}\right) \Gamma\left(s+\frac{l}{2}+\frac{1}{2}-\frac{\sqrt{-1} r}{2}\right) \\
& \times \zeta(2 s) \sum_{m=1}^{\infty} \frac{c(-m) b(2 m)}{m^{s+l / 2}} .
\end{aligned}
$$

We put $\quad \Gamma_{l, r}(s):=\pi^{-(2 s+l+1)} \Gamma\left(s+l / 2+\frac{1}{2}+\sqrt{-1} r / 2\right) \Gamma\left(s+l / 2+\frac{1}{2}-\right.$ $\sqrt{-1} r / 2)$. We have

$$
\begin{aligned}
& \xi\left(s+\frac{l}{2}+\frac{1}{2}, P_{l, \nu}\right)=\Gamma_{l, r}(s) \sum_{\beta \in S \backslash\{0\}} \frac{A(\beta) P_{l, \nu}(\beta)}{|\beta|^{2(s+l / 2+1 / 2)}} \\
& =\Gamma_{l, r}(s) \sum_{\beta \in S \backslash\{0\}} \frac{\sum_{t=0}^{u} \sum_{n \mid d} c\left(\frac{-|\beta|^{2}}{2^{t+1} n^{2}}\right)(-\epsilon)^{t} P_{l, \nu}(\beta)}{\left(|\beta|^{2}\right)^{s+l / 2}} \\
& =\Gamma_{l, r}(s) \sum_{\beta \in S \backslash\{0\}} \sum_{t=0}^{u} \sum_{n \mid d} \frac{c\left(-\frac{|\beta|^{2}}{2^{t+1} n^{2}}\right)(-\epsilon)^{t} P_{l, \nu}\left(\frac{\beta}{2^{t / 2} n}\right)}{\left(2^{t} n^{2}\right)^{s}\left(\frac{|\beta|^{2}}{2^{t} n^{2}}\right)^{s+l / 2}} \\
& =\Gamma_{l, r}(s) \sum_{u=0}^{\infty} \sum_{\substack{d \geqslant 1 \\
d: \text { odd }}} \sum_{\beta \in S_{\text {prim }}} \sum_{t=0}^{u} \sum_{n \mid d} \\
& \times \frac{c\left(-\frac{1}{2}\left|\varpi_{2}^{u-t} \frac{d}{n} \beta_{0}\right|^{2}\right)\left(-\epsilon \epsilon_{l, \nu}\right)^{t} P_{l, \nu}\left(\varpi_{2}^{u-t} \frac{d}{n} \beta_{0}\right)}{\left(2^{t} n^{2}\right)^{s}\left|\varpi_{2}^{u-t} \frac{d}{n} \beta_{0}\right|^{2(s+l / 2)}} \\
& =\Gamma_{l, r}(s) \sum_{u=0}^{\infty} \\
& \times \sum_{\substack{d \geqslant 1 \\
d: \text { odd }}}\left(\sum_{t=0}^{u} \sum_{n \mid d} \sum_{\beta \in S^{\text {prim }}} \frac{c\left(-\frac{1}{2}\left|\varpi_{2}^{t} n \beta\right|^{2}\right) P_{l, \nu}\left(\varpi_{2}^{t} n \beta\right)}{\left(-2^{s} \epsilon_{l, \nu} \epsilon\right)^{u-t}\left(\frac{d}{n}\right)^{2 s}\left|\varpi_{2}^{t} n \beta\right|^{2(s+l / 2)}}\right) \\
& =\Gamma_{l, r}(s) \sum_{u=0}^{\infty} \frac{1}{\left(-2^{s} \epsilon_{l, \nu} \epsilon\right)^{u}} \sum_{\substack{d \geqslant 1 \\
d: \text { odd }}} \frac{1}{d^{2 s}} \\
& \times \sum_{m=1}^{\infty} \frac{c(-m) \sum_{\beta \in S,|\beta|^{2}=2 m} P_{l, \nu}(\beta)}{(2 m)^{s+l / 2}} \\
& =2^{1-l / 2} \frac{2^{2 s}-1}{2^{s}+\epsilon_{l, \nu} \epsilon} 2^{-2 s-1} \Gamma_{l, r}(s) \zeta(2 s) \sum_{m=1}^{\infty} \frac{c(-m) b(2 m)}{m^{s+l / 2}} \\
& =2^{1-l / 2} \pi^{-(l+1)}\left(2^{s}-\epsilon_{l, \nu} \epsilon\right) I(s) .
\end{aligned}
$$


We note that $\xi\left(s, \hat{P}_{l, \nu}\right)=\xi\left(s, P_{l, \nu}\right)$ since $S=\bar{S}$. The formula just proved and Theorem 3.3 then imply that $\xi\left(s, P_{l, \nu}\right)$ satisfies the desired three analytic properties in Theorem 4.1.

REMARK 4.3. Note that the definition (4.2) of $A(\beta)$ is very similar to the formula for the Fourier coefficient of the lift in the Saito-Kurokawa case (see [7, page 348]) and the GSpin $(1,4)$ case $[22,(3.4)]$.

THEOREM 4.4. Let $f \in S\left(\Gamma_{0}(2) ;-\left(\frac{1}{4}+r^{2} / 4\right)\right)$ with Fourier coefficients $c(n)$ and with eigenvalue $\epsilon$ of the Atkin-Lehner involution. Define

$$
F_{f}\left(n(x) a_{y}\right):=\sum_{\beta \in S \backslash\{0\}} A(\beta) y^{2} K_{\sqrt{-1} r}(2 \pi|\beta| y) e^{2 \pi \sqrt{-1} \operatorname{Re}(\beta x)}
$$

with $\{A(\beta)\}_{\beta \in S \backslash\{0\}}$ defined by (4.2). Then we have $F_{f} \in \mathcal{M}\left(\mathrm{GL}_{2}(\mathcal{O}) ; r\right)$ and $F_{f}$ is a cusp form. Furthermore, $F_{f} \not \equiv 0$ if $f \not \equiv 0$.

Proof. We can verify the left invariance of $F_{f}$ with respect to $\left\{\left[\begin{array}{ll}u & 0 \\ 0 & 1\end{array}\right] \mid u \in \mathcal{O}^{\times}\right\}$in a straightforward way. Proposition 2.2, Theorem 4.1 and Proposition 4.2 thus imply $F_{f} \in \mathcal{M}\left(G L_{2}(\mathcal{O}) ; r\right)$. Since $\mathrm{GL}_{2}(\mathcal{O})$ has only one cusp (see Lemma 2.3), the Fourier expansion of $F_{f}$ means that $F_{f}$ is cuspidal. To show the nonvanishing we need the following lemma.

LEMmA 4.5. Let $f \in S\left(\Gamma_{0}(2) ;-\left(\frac{1}{4}+r^{2} / 4\right)\right) \backslash\{0\}$ with Fourier coefficients $c(n)$ and with eigenvalue $\epsilon$ of the Atkin-Lehner involution. Then, there exist $N>0, N \in \mathbb{Z}$, such that $c(-N) \neq 0$.

Proof. Assume that $c(n)=0$ for all $n<0$. Set $f_{1}(z)=(f(z)+f(-\bar{z})) / 2$ and $f_{2}(z)=(f(z)-f(-\bar{z})) / 2$. Then, $f_{1}, f_{2}$ are elements of $S\left(\Gamma_{0}(2) ;-\left(\frac{1}{4}+\right.\right.$ $\left.r^{2} / 4\right)$ ) with the same eigenvalue $\epsilon$ of the Atkin-Lehner involution as $f$. In addition, $f_{1}$ is an even Maass form and $f_{2}$ is an odd Maass form, with the property that they have the exact same Fourier coefficients corresponding to positive indices. This implies that the $L$-functions for $f_{1}$ and $f_{2}$ satisfy $L\left(s, f_{1}\right)=L\left(s, f_{2}\right)$. On the other hand, $L\left(s, f_{1}\right)$ and $L\left(s, f_{2}\right)$ satisfy functional equations with the gamma factors shifted by 1 . Here, we use that both $f_{1}$ and $f_{2}$ have the same Atkin-Lehner eigenvalue. If $L\left(s, f_{1}\right) \neq 0$, we obtain an identity of gamma factors, which can be checked to be impossible. This gives us that $f$ has to be zero, a contradiction.

Let $N_{0}$ be the smallest positive integer such that $c\left(-N_{0}\right) \neq 0$. Let $\beta_{0} \in \mathcal{O}$ be such that $\left|\beta_{0}\right|^{2}=N_{0}$. Choose $\beta=\varpi_{2} \beta_{0}$. Then, by the choice of $N_{0}$ and the definition of $A(\beta)$, we see that $A(\beta)=\sqrt{2 N_{0}} c\left(-N_{0}\right) \neq 0$, as required. 
REMARK 4.6. Weyl's law for congruence subgroups of $\mathrm{SL}_{2}(\mathbb{Z})$ by Selberg (cf. $[12, \S 11.1]$ ) implies that there exist Maass cusp forms for $\Gamma_{0}(2)$. This and the theorem above imply the existence of nonzero lifts $F_{f}$.

\section{$\S 5$. Actions of Hecke operators on the lifting}

\subsection{Adelization of automorphic forms}

To study the actions of Hecke operators on our cusp forms constructed by the lifting we need both adelic and nonadelic treatments of automorphic forms.

For a complex number $r \in \mathbb{C}$ we introduce another space $M(\mathcal{G}(\mathbb{A}), r)$ of automorphic forms for $\mathcal{G}$.

Definition 5.1. Let $M(\mathcal{G}(\mathbb{A}), r)$ be the space of smooth functions $\Phi$ on $\mathcal{G}(\mathbb{A})$ satisfying the following conditions:

(1) $\Phi\left(z \gamma g u_{f} u_{\infty}\right)=\Phi(g)$ for any $\left(z, \gamma, g, u_{f}, u_{\infty}\right) \in Z_{\mathbb{A}} \times \mathcal{G}(\mathbb{Q}) \times \mathcal{G}(\mathbb{A}) \times$

$U \times K$, where $Z_{\mathbb{A}}$ denotes the center of $\mathcal{G}(\mathbb{A})$;

(2) $\Omega \cdot \Phi\left(g_{\infty}\right)=-\frac{1}{2}\left(r^{2} / 4+1\right) \Phi\left(g_{\infty}\right)$ for any $g_{\infty} \in \mathcal{G}(\mathbb{R})=\mathrm{GL}_{2}(\mathbb{H})$;

(3) $\Phi$ is of moderate growth.

According to part (1) of Lemma 2.3, the class number of $\mathcal{G}$ with respect to $U$ is one, which means that $\mathcal{G}(\mathbb{A})=\mathcal{G}(\mathbb{Q}) \mathcal{G}(\mathbb{R}) U$. We can thus view $F \in$ $M\left(\mathrm{GL}_{2}(\mathcal{O}), r\right)$ as a smooth function $\Phi_{F}$ on $\mathcal{G}(\mathbb{A})$ by

$$
\Phi_{F}\left(\gamma g_{\infty} u_{f}\right)=F\left(g_{\infty}\right) \quad \forall\left(\gamma, g_{\infty}, u_{f}\right) \in \mathcal{G}(\mathbb{Q}) \times \mathcal{G}(\mathbb{R}) \times U
$$

We therefore see the following.

LEMMA 5.2. We have an isomorphism $M\left(\mathrm{GL}_{2}(\mathcal{O}), r\right) \simeq M(\mathcal{G}(\mathbb{A}), r)$.

\subsection{Hecke operators}

For each place $p \leqslant \infty$, let $\mathcal{G}_{p}:=\mathrm{GL}_{2}\left(B_{p}\right)$, with $B_{p}=B \otimes_{\mathbb{Q}} \mathbb{Q}_{p}$. For a finite prime $p \neq 2$, we have $\mathrm{GL}_{2}\left(B_{p}\right) \simeq \mathrm{GL}_{4}\left(\mathbb{Q}_{p}\right)$. Let $\mathcal{O}_{p}$ be the $p$-adic completion of $\mathcal{O}$ for $p<\infty$. For a finite prime $p \neq 2, \mathcal{O}_{p} \simeq M_{2}\left(\mathbb{Z}_{p}\right)$ and $\mathrm{GL}_{2}\left(\mathcal{O}_{p}\right) \simeq \mathrm{GL}_{4}\left(\mathbb{Z}_{p}\right)$. Set $K_{p}=\mathrm{GL}_{2}\left(\mathcal{O}_{p}\right)$ for $p<\infty$.

We denote by $\mathcal{H}_{p}=\mathcal{H}\left(\mathcal{G}_{p}, K_{p}\right)$ the Hecke algebra for $\operatorname{GL}_{2}\left(B_{p}\right)$ with respect to $\mathrm{GL}_{2}\left(\mathcal{O}_{p}\right)$ for $p<\infty$. According to [26, $\S 8$, Theorem 6$], \mathcal{H}_{p}$ has the following generators:

$$
\begin{cases}\left\{\varphi_{1}^{ \pm 1}, \varphi_{2}\right\} & \text { if } p=2 \\ \left\{\phi_{1}^{ \pm 1}, \phi_{2}, \phi_{3}, \phi_{4}\right\} & \text { if } p \neq 2\end{cases}
$$


Here, $\varphi_{1}, \varphi_{2}$ denote the characteristic functions for

$$
K_{2}\left[\begin{array}{cc}
\varpi_{2} & 0 \\
0 & \varpi_{2}
\end{array}\right] K_{2}, \quad K_{2}\left[\begin{array}{cc}
\varpi_{2} & 0 \\
0 & 1
\end{array}\right] K_{2}
$$

respectively, and $\phi_{1}, \phi_{2}, \phi_{3}, \phi_{4}$ denote the characteristic functions for

$$
\begin{gathered}
K_{p}\left[\begin{array}{llll}
p & & & \\
& p & & \\
& & p & \\
& & & p
\end{array}\right] K_{p}, K_{p}\left[\begin{array}{llll}
p & & & \\
& p & & \\
& & p & \\
& & & 1
\end{array}\right] K_{p}, \\
K_{p}\left[\begin{array}{llll}
p & & & \\
& p & & \\
& & 1 & \\
& & & 1
\end{array}\right] K_{p}, K_{p}\left[\begin{array}{llll}
p & & & \\
& 1 & & \\
& & 1 & \\
& & & 1
\end{array}\right] K_{p}
\end{gathered}
$$

respectively when $p \neq 2$. Recall that $\varpi_{2}$ denotes a prime element of $B_{2}$. We want to obtain the single coset decomposition for the above double cosets. For that, we next review the Bruhat decomposition of $K_{p}$ given by

$$
K_{p}=\bigsqcup_{w \in W_{p}} T_{p} w T_{p},
$$

where $W_{p}$ denotes the Weyl group of $\mathrm{GL}_{2}\left(B_{p}\right)$, and $T_{p}$ is the subgroup of elements in $K_{p}$ which are upper triangular modulo $p$ for an odd $p$ (respectively, upper triangular modulo $\varpi_{2} \mathcal{O}_{2}$ for $p=2$ ).

Let $N_{p}$ be the standard maximal unipotent subgroup of $\mathrm{GL}_{2}\left(B_{p}\right)$ defined over $\mathbb{Q}_{p}$. We put $N_{p}^{0}\left(\mathbb{Z}_{p}\right):=T_{p} \cap{ }^{t} N_{p}\left(\mathbb{Q}_{p}\right)$. We furthermore introduce $N\left(\mathbb{Z}_{p}\right)=N_{p}\left(\mathbb{Q}_{p}\right) \cap K_{p}$ and $D\left(\mathbb{Z}_{p}\right)=D_{p}\left(\mathbb{Q}_{p}\right) \cap K_{p}$, where $D_{p}$ denotes the subgroup of diagonal matrices in $\mathrm{GL}_{2}\left(B_{p}\right)$. Then we have the Iwahori decomposition

$$
T_{p}=N\left(\mathbb{Z}_{p}\right) D\left(\mathbb{Z}_{p}\right) N_{p}^{0}\left(\mathbb{Z}_{p}\right)
$$

(see [13, Theorem 2.5]). Let $h$ be one of

$$
\begin{gathered}
1_{4}\left(\text { or } 1_{2}\right), \quad\left[\begin{array}{llll}
p & & & \\
& p & & \\
& & p & \\
& & p
\end{array}\right], \quad\left[\begin{array}{llll}
p & & \\
& p & & \\
& & p & \\
& & & 1
\end{array}\right], \\
{\left[\begin{array}{llll}
p & & & \\
& p & & \\
& & 1 & \\
& & 1
\end{array}\right], \quad\left[\begin{array}{llll}
p & & & \\
& 1 & & \\
& & 1 & \\
& & & 1
\end{array}\right] \text { or }\left[\begin{array}{lll}
\varpi_{2} & \\
& 1
\end{array}\right] .}
\end{gathered}
$$


LEMMA 5.3.

$$
K_{p} h K_{p}=\bigsqcup_{w \in W_{p} / W_{p}(h)} N\left(\mathbb{Z}_{p}\right) w h K_{p}
$$

where $W_{p}(h):=\left\{w \in W_{p} \mid w h w^{-1}=h\right\}$.

To describe this coset decomposition of $K_{p} h K_{p}$ explicitly, we need a set of representatives for $W_{p} / W_{p}(h)$.

LEMMA 5.4.

(1) Let $p=2$. For $h=\left[\begin{array}{cc}\varpi_{2} & 0 \\ 0 & 1\end{array}\right]$ we can take

$$
\left\{1_{2}, \quad\left[\begin{array}{ll}
0 & 1 \\
1 & 0
\end{array}\right]\right\}
$$

as a set of representatives for $W_{2} / W_{2}(h)$.

(2) Let $p \neq 2$.

(a) When $h=\left[\begin{array}{lll}p & & \\ & p & \\ & p & \\ & & 1\end{array}\right]$ we can take

$$
\left\{1_{4},\left[\begin{array}{llll}
0 & 0 & 0 & 1 \\
0 & 1 & 0 & 0 \\
0 & 0 & 1 & 0 \\
1 & 0 & 0 & 0
\end{array}\right],\left[\begin{array}{llll}
1 & 0 & 0 & 0 \\
0 & 0 & 0 & 1 \\
0 & 0 & 1 & 0 \\
0 & 1 & 0 & 0
\end{array}\right],\left[\begin{array}{llll}
1 & 0 & 0 & 0 \\
0 & 1 & 0 & 0 \\
0 & 0 & 0 & 1 \\
0 & 0 & 1 & 0
\end{array}\right]\right\}
$$

as a set of representatives for $W_{p} / W_{p}(h)$.

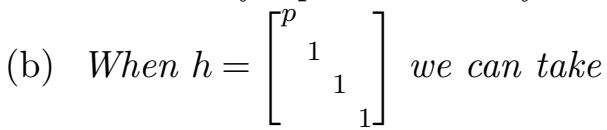

$$
\left\{1_{4},\left[\begin{array}{llll}
0 & 0 & 0 & 1 \\
0 & 1 & 0 & 0 \\
0 & 0 & 1 & 0 \\
1 & 0 & 0 & 0
\end{array}\right],\left[\begin{array}{llll}
0 & 0 & 1 & 0 \\
0 & 1 & 0 & 0 \\
1 & 0 & 0 & 0 \\
0 & 0 & 0 & 1
\end{array}\right],\left[\begin{array}{llll}
0 & 1 & 0 & 0 \\
1 & 0 & 0 & 0 \\
0 & 0 & 1 & 0 \\
0 & 0 & 0 & 1
\end{array}\right]\right\}
$$

as a set of representatives for $W_{p} / W_{p}(h)$. 
(c) When $h=\left[\begin{array}{lll}p & & \\ & p & \\ & 1 & \\ & & 1\end{array}\right]$ we can take

$$
\begin{gathered}
1_{4},\left[\begin{array}{llll}
0 & 0 & 1 & 0 \\
0 & 1 & 0 & 0 \\
1 & 0 & 0 & 0 \\
0 & 0 & 0 & 1
\end{array}\right],\left[\begin{array}{llll}
0 & 0 & 0 & 1 \\
0 & 1 & 0 & 0 \\
0 & 0 & 1 & 0 \\
1 & 0 & 0 & 0
\end{array}\right],\left[\begin{array}{llll}
1 & 0 & 0 & 0 \\
0 & 0 & 1 & 0 \\
0 & 1 & 0 & 0 \\
0 & 0 & 0 & 1
\end{array}\right], \\
\left.\left[\begin{array}{llll}
1 & 0 & 0 & 0 \\
0 & 0 & 0 & 1 \\
0 & 0 & 1 & 0 \\
0 & 1 & 0 & 0
\end{array}\right],\left[\begin{array}{llll}
0 & 0 & 1 & 0 \\
0 & 0 & 0 & 1 \\
1 & 0 & 0 & 0 \\
0 & 1 & 0 & 0
\end{array}\right]\right\}
\end{gathered}
$$

as a set of representatives for $W_{p} / W_{p}(h)$.

By Lemmas 5.3 and 5.4, we are now able to write down the coset decomposition of $K_{p} h K_{p}$ explicitly.

LEMMA 5.5.

(1) Let $p=2$ and $h=\left[\begin{array}{cc}\varpi_{2} & 0 \\ 0 & 1\end{array}\right]$. We have

$$
K_{2} h K_{2}=\left[\begin{array}{cc}
1 & 0 \\
0 & \varpi_{2}
\end{array}\right] K_{2} \sqcup \underset{x \in \mathcal{O}_{2} / \varpi_{2} \mathcal{O}_{2}}{\bigsqcup}\left[\begin{array}{cc}
\varpi_{2} & 0 \\
0 & 1
\end{array}\right]\left[\begin{array}{cc}
1 & \varpi_{2}^{-1} x \\
0 & 1
\end{array}\right] K_{2} .
$$

(2) Let $p \neq 2$.

(a) For $h=\left[\begin{array}{lll}p & & \\ & p & \\ & p & \\ & & \end{array}\right]$ we have

$$
\begin{aligned}
& K_{p} h K_{p}=\bigsqcup_{x_{14}, x_{24}, x_{34} \in \mathbb{Z}_{p} / p \mathbb{Z}_{p}}\left[\begin{array}{llll}
p & & & \\
& p & & \\
& p & x_{34} \\
& & 1
\end{array}\right]\left[\begin{array}{cccc}
1 & & & p^{-1} x_{14} \\
& 1 & & p^{-1} x_{24} \\
& 1 & \\
& & & 1
\end{array}\right] K_{p} \\
& \sqcup \bigsqcup_{x_{12} \in \mathbb{Z}_{p} / p \mathbb{Z}_{p}}\left[\begin{array}{cccc}
p & x_{12} & & \\
& 1 & & \\
& & p & \\
& & & p
\end{array}\right] K_{p} \\
& \sqcup \bigsqcup_{x_{13}, x_{23} \in \mathbb{Z}_{p} / p \mathbb{Z}_{p}}\left[\begin{array}{llll}
p & & & \\
& p & & \\
& & 1 & \\
& & & p
\end{array}\right]\left[\begin{array}{cccc}
1 & & p^{-1} x_{13} & \\
& 1 & p^{-1} x_{23} & \\
& & 1 & \\
& & & 1
\end{array}\right] K_{p}
\end{aligned}
$$




$$
\sqcup\left[\begin{array}{llll}
1 & & & \\
& p & & \\
& & p & \\
& & & p
\end{array}\right] K_{p}
$$

(b) For $h=\left[\begin{array}{llll}p & & \\ & 1 & & \\ & & 1 \\ & & & 1\end{array}\right]$ we have

$$
\begin{aligned}
& K_{p} h K_{p}=\bigsqcup_{x_{12}, x_{13}, x_{14} \in \mathbb{Z}_{p} / p \mathbb{Z}_{p}}\left[\begin{array}{cccc}
p & x_{12} & & \\
& 1 & & \\
& & 1 & \\
& & & 1
\end{array}\right] \\
& \times\left[\begin{array}{cccc}
1 & & p^{-1} x_{13} & p^{-1} x_{14} \\
& 1 & 1 & \\
& & & 1
\end{array}\right] K_{p} \\
& \sqcup \bigsqcup_{x_{34} \in \mathbb{Z}_{p} / p \mathbb{Z}_{p}}\left[\begin{array}{cccc}
1 & & & \\
& 1 & & \\
& & p & x_{34} \\
& & & 1
\end{array}\right] K_{p} \\
& \sqcup \bigsqcup_{x_{23}, x_{24} \in \mathbb{Z}_{p} / p \mathbb{Z}_{p}}\left[\begin{array}{cccc}
1 & & & \\
& p & & \\
& & 1 & \\
& & & 1
\end{array}\right]\left[\begin{array}{cccc}
1 & & & \\
& 1 & p^{-1} x_{23} & p^{-1} x_{24} \\
& & 1 & \\
& & & 1
\end{array}\right] K_{p} \\
& \sqcup\left[\begin{array}{llll}
1 & & & \\
& 1 & & \\
& & 1 & \\
& & & p
\end{array}\right] K_{p}
\end{aligned}
$$

(c) For $h=\left[\begin{array}{lll}p & & \\ & p & \\ & & 1 \\ & & 1\end{array}\right]$ we have

$$
K_{p} h K_{p}=\bigsqcup_{x_{13}, x_{14}, x_{23}, x_{24} \in \mathbb{Z}_{p} / p \mathbb{Z}_{p}}\left[\begin{array}{llll}
p & & & \\
& p & & \\
& & 1 & \\
& & & 1
\end{array}\right]
$$




$$
\begin{aligned}
& \times\left[\begin{array}{cccc}
1 & & p^{-1} x_{13} & p^{-1} x_{14} \\
& 1 & p^{-1} x_{23} & p^{-1} x_{24} \\
& & 1 & \\
& & & 1
\end{array}\right] K_{p} \\
& \sqcup \bigsqcup_{x_{24}, x_{34} \in \mathbb{Z}_{p} / p \mathbb{Z}_{p}}\left[\begin{array}{cccc}
1 & & & \\
& p & & \\
& & p & x_{34} \\
& & & 1
\end{array}\right]\left[\begin{array}{cccc}
1 & & & \\
& 1 & & p^{-1} x_{24} \\
& & 1 & \\
& & & 1
\end{array}\right] K_{p} \\
& \sqcup \bigsqcup_{x_{23} \in \mathbb{Z}_{p} / p \mathbb{Z}_{p}}\left[\begin{array}{llll}
1 & & & \\
& p & & \\
& & 1 & \\
& & & p
\end{array}\right]\left[\begin{array}{cccc}
1 & & & \\
& 1 & p^{-1} x_{23} & \\
& & 1 & \\
& & & 1
\end{array}\right] K_{p} \\
& \sqcup \bigsqcup_{x_{12}, x_{14}, x_{34} \in \mathbb{Z}_{p} / p \mathbb{Z}_{p}}\left[\begin{array}{cccc}
p & x_{12} & & \\
& 1 & & \\
& & p & x_{34} \\
& & & 1
\end{array}\right]\left[\begin{array}{cccc}
1 & & & p^{-1} x_{14} \\
& 1 & & \\
& & 1 & \\
& & & 1
\end{array}\right] K_{p} \\
& \sqcup \bigsqcup_{x_{12}, x_{13} \in \mathbb{Z}_{p} / p \mathbb{Z}_{p}}\left[\begin{array}{cccc}
p & x_{12} & & \\
& 1 & & \\
& & 1 & \\
& & & p
\end{array}\right]\left[\begin{array}{cccc}
1 & & p^{-1} x_{13} & \\
& 1 & & \\
& & 1 & \\
& & & 1
\end{array}\right] K_{p} \\
& \sqcup\left[\begin{array}{llll}
1 & & & \\
& 1 & & \\
& & p & \\
& & & p
\end{array}\right] K_{p} .
\end{aligned}
$$

We can now describe the actions of the Hecke operators defined by the $K_{p} h K_{p}$ s above. With the invariant measure $d x$ of $\mathcal{G}_{p}$ normalized so that $\int_{K_{p}} d x=1$, we define $K_{p} h K_{p} \cdot \Phi$ by

$$
\left(K_{p} h K_{p} \cdot \Phi\right)(g):=\int_{\mathcal{G}_{p}} \operatorname{char}_{K_{p} h K_{p}}(x) \Phi(g x) d x
$$

for $\Phi \in M(\mathcal{G}(\mathbb{A}), r)$, where $\operatorname{char}_{K_{p} h K_{p}}$ denotes the characteristic function for $K_{p} h K_{p}$.

We provide the nonadelic description of $K_{p} h K_{p} \cdot \Phi$, which enables us to describe explicitly the influence of the $K_{p} h K_{p}$-action on Fourier coefficients of the lifting $F_{f}$. 
To this end we now introduce the sets

$C_{p}:=\{\alpha \in \mathcal{O} \mid \nu(\alpha)=p\} / \mathcal{O}^{\times}, \quad C_{p}^{\prime}:=\left\{x \in M_{2}\left(\mathbb{Z}_{p}\right) \mid \operatorname{det}(x)=p\right\} / G L_{2}\left(\mathbb{Z}_{p}\right)$,

and state the following lemma.

LEMMA 5.6.

(1) There is a bijection

$$
C_{p}^{\prime} \simeq\left\{\left[\begin{array}{ll}
1 & 0 \\
0 & p
\end{array}\right], \quad\left[\begin{array}{ll}
p & b \\
0 & 1
\end{array}\right] \mid b \in \mathbb{Z}_{p} / p \mathbb{Z}_{p}\right\} .
$$

(2) For an odd prime $p$, the isomorphism $\mathcal{O}_{p} \simeq M_{2}\left(\mathbb{Z}_{p}\right)$ induces the bijection

$$
C_{p} \simeq C_{p}^{\prime}
$$

Proof. The first assertion is verified by a direct calculation. We prove the second assertion. As is remarked in the proof of [22, Proposition 5.2], we have $\# C_{p}=p+1$. Under the isomorphism $\mathcal{O}_{p} \simeq M_{2}\left(\mathbb{Z}_{p}\right)$, we can regard any element in $\mathcal{O}$ as one lying in $M_{2}\left(\mathbb{Z}_{p}\right)$. Any two inequivalent representatives of $C_{p}$ are not equivalent to each other in $\left\{x \in M_{2}\left(\mathbb{Z}_{p}\right) \mid \operatorname{det}(x)=p\right\} / \mathrm{GL}_{2}\left(\mathbb{Z}_{p}\right)$. Otherwise there are two inequivalent representatives $\alpha_{1}$ and $\alpha_{2}$ of $C_{p}$ which are equivalent under $\mathcal{O}_{l}^{\times}$-action for all primes $l$, since $\alpha_{1} / \alpha_{2} \in \mathcal{O}_{l}^{\times}$for all primes $l$. Note here that $\mathcal{O}_{l}^{\times} \simeq \mathrm{GL}_{2}\left(\mathbb{Z}_{l}\right)$ for any odd primes $l$. This, however, implies that two such representatives are equivalent to each other in $C_{p}$. We therefore know that there is a injection from $C_{p}$ into $\left\{x \in M_{2}\left(\mathbb{Z}_{p}\right) \mid \operatorname{det}(x)=\right.$ $p\} / \mathrm{GL}_{2}\left(\mathbb{Z}_{p}\right)$. Since the latter set also has $p+1$ representatives as in the statement, the injection is actually a bijection.

Let $F \in M\left(\mathrm{GL}_{2}(\mathcal{O}), r\right)$ correspond to $\Phi$. By $K_{p} h K_{p} \cdot F$ we denote the cusp form in $M\left(\mathrm{GL}_{2}(\mathcal{O}), r\right)$ corresponding to $K_{p} h K_{p} \cdot \Phi$. Due to Lemma 2.3, $\mathcal{G}(\mathbb{Q}) \backslash \mathcal{G}(\mathbb{A}) / U$ has a complete set of representatives in $\mathcal{G}(\mathbb{R})$, where $\mathcal{G}(\mathbb{R})$ is viewed as a subgroup of $\mathcal{G}(\mathbb{A})$ in the usual manner. The cusp form $K_{p} h K_{p} \cdot \Phi$ is thereby determined by its restriction to $\mathcal{G}(\mathbb{R})$, which is nothing but $K_{p} h K_{p} \cdot F$. Moreover, we remark that an element in $M\left(\mathrm{GL}_{2}(\mathcal{O}), r\right)$ is determined by its restriction to $N A=\left\{n(x) a_{y} \mid x \in \mathbb{H}, y \in \mathbb{R}_{+}^{\times}\right\}$(see (2.1)).

Proposition 5.7. Let $K_{p} h K_{p} \cdot F$ be as above, and let $n(x)$ and $a_{y}$ be as defined in (2.1). For $p$ odd, let $C_{p}$ be as defined in (5.3). 
(1) Let $p=2$, and let $h=\left[\begin{array}{cc}\varpi_{2} & 0 \\ 0 & 1\end{array}\right]$. We have

$$
\left(K_{2} h K_{2} \cdot F\right)\left(n(x) a_{y}\right)=F\left(n\left(\varpi_{2} x\right) a_{2^{1 / 2} y}\right)+2^{2} F\left(n\left(\varpi_{2}^{-1} x\right) a_{2^{-1 / 2} y}\right) .
$$

(2) Let $p \neq 2$.

(a) When $h=\left[\begin{array}{lll}p & & \\ & p & \\ & p \\ & & 1\end{array}\right]$, we have

$$
\begin{aligned}
\left(K_{p} h K_{p} \cdot F\right)\left(n(x) a_{y}\right)= & \sum_{\alpha \in C_{p}} F\left(n(\alpha x) a_{p^{1 / 2} y}\right) \\
& +p^{2} \sum_{\alpha \in C_{p}} F\left(n\left(x \alpha^{-1}\right) a_{p^{-1 / 2} y}\right) .
\end{aligned}
$$

(b) When $h=\left[\begin{array}{llll}p & & & \\ & 1 & & \\ & & 1 & \\ & & & 1\end{array}\right]$, we have

$$
\begin{aligned}
\left(K_{p} h K_{p} \cdot F\right)\left(n(x) a_{y}\right)= & \sum_{\alpha \in C_{p}} F\left(n(x \alpha) a_{p^{1 / 2} y}\right) \\
& +p^{2} \sum_{\alpha \in C_{p}} F\left(n\left(\alpha^{-1} x\right) a_{p^{-1 / 2} y}\right) .
\end{aligned}
$$

(c) When $h=\left[\begin{array}{lll}p & & \\ & p & \\ & 1 \\ & & 1\end{array}\right]$, we have

$$
\begin{aligned}
\left(K_{p} h K_{p} \cdot F\right)\left(n(x) a_{y}\right)= & F\left(n(p x) a_{p y}\right)+p^{4} F\left(n\left(p^{-1} x\right) a_{p^{-1} y}\right) \\
& +p \sum_{\left(\alpha_{1}, \alpha_{2}\right) \in C_{p} \times C_{p}} F\left(n\left(\alpha_{1}^{-1} x \alpha_{2}\right) a_{y}\right) .
\end{aligned}
$$

Proof. We prove only (2c). The other cases are settled similarly. We make use of the isomorphism $\psi: \mathcal{O}_{p} \rightarrow M_{2}\left(\mathbb{Z}_{p}\right)$, given explicitly as follows. Since $p$ is odd, we can find $r, s \in \mathbb{Z}_{p}$ such that $1+r^{2}+s^{2}=0$. Then, we have

$$
\psi\left(a_{0}+a_{1} i+a_{2} j+a_{3} i j\right)=\left[\begin{array}{cc}
a_{0}-a_{1} r-a_{2} s & -a_{3}-a_{1} s+a_{2} r \\
a_{3}-a_{1} s+a_{2} r & a_{0}+a_{1} r+a_{2} s
\end{array}\right] .
$$

Let $x_{1}, x_{2}, x_{3}, x_{4} \in \mathcal{O}$ such that

$$
\psi\left(x_{1}\right) \in\left[\begin{array}{ll}
1 & 0 \\
0 & 0
\end{array}\right]+M_{2}\left(p \mathbb{Z}_{p}\right), \quad \psi\left(x_{2}\right) \in\left[\begin{array}{ll}
0 & 1 \\
0 & 0
\end{array}\right]+M_{2}\left(p \mathbb{Z}_{p}\right),
$$




$$
\psi\left(x_{3}\right) \in\left[\begin{array}{ll}
0 & 0 \\
1 & 0
\end{array}\right]+M_{2}\left(p \mathbb{Z}_{p}\right), \quad \psi\left(x_{4}\right) \in\left[\begin{array}{ll}
0 & 0 \\
0 & 1
\end{array}\right]+M_{2}\left(p \mathbb{Z}_{p}\right) .
$$

For example, let $r_{0}, s_{0} \in\{0,1, \ldots, p-1\}$ such that $r-r_{0}, s-s_{0} \in p \mathbb{Z}_{p}$. Then, we can choose $x_{4}=\frac{p+1}{2}\left(1-r_{0} i-s_{0} j\right)$, where note that $\frac{p+1}{2} \in \mathbb{Z}$ since $p$ is odd. Denote by $\alpha_{0}, \alpha_{1}, \alpha_{2}, \ldots, \alpha_{p}$ the representatives of $C_{p}$ such that

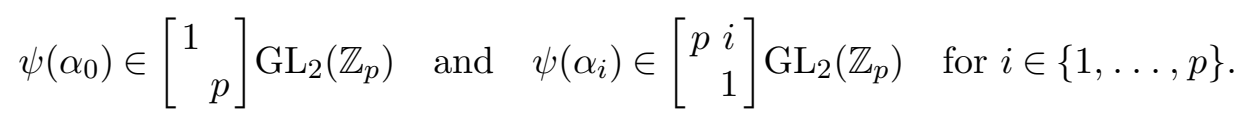

The left coset decomposition of $K_{p} h K_{p}$ in part (2c) of Lemma 5.5 can be rewritten as

$$
\begin{aligned}
& K_{p} h K_{p}=\bigsqcup_{x \in \mathcal{O} / p \mathcal{O}}\left[\begin{array}{rr}
\psi(1) & \psi(x) \\
& \psi(1)
\end{array}\right]\left[\begin{array}{ll}
\psi(p) & \\
& \psi(1)
\end{array}\right] K_{p} \\
& \sqcup \bigsqcup_{i, t \in\{1, \ldots, p\}}\left[\begin{array}{r}
\psi(1) t \psi\left(x_{4}\right) \\
\psi(1)
\end{array}\right]\left[\begin{array}{ll}
\psi\left(\alpha_{0}\right) & \\
& \psi\left(\alpha_{i}\right)
\end{array}\right] K_{p}
\end{aligned}
$$

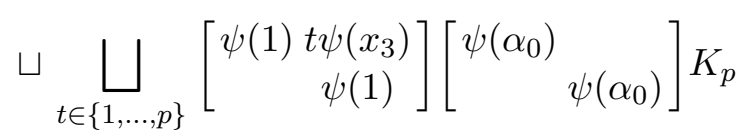

$$
\begin{aligned}
& \sqcup \bigsqcup_{i, j, t \in\{1, \ldots, p\}}\left[\begin{array}{r}
\psi(1) t \psi\left(x_{2}\right) \\
\psi(1)
\end{array}\right]\left[\begin{array}{rl}
\psi\left(\alpha_{i}\right) & \\
& \psi\left(\alpha_{j}\right)
\end{array}\right] K_{p} \\
& \sqcup \bigsqcup_{i, t \in\{1, \ldots, p\}}\left[\begin{array}{r}
\psi(1) t \psi\left(x_{1}\right) \\
\psi(1)
\end{array}\right]\left[\begin{array}{rl}
\psi\left(\alpha_{i}\right) & \\
& \psi\left(\alpha_{0}\right)
\end{array}\right] K_{p} \\
& \sqcup\left[\begin{array}{ll}
\psi(1) & \\
& \psi(p)
\end{array}\right] K_{p} .
\end{aligned}
$$

We note that $\left(K_{p} h K_{p} \Phi\right)\left(\left(n(x) a_{y}\right)_{\infty} \prod_{v<\infty} 1_{v}\right)$ is the sum of terms of the form $\Phi\left(\left(n(x) a_{y}\right)_{\infty} \cdot \gamma_{p} \cdot \prod_{v \neq p, \infty} 1_{v}\right)$, where $\gamma_{p}$ runs over the single coset representatives above. Since we have chosen the coset representatives $\gamma_{p}$ above in $M_{2}(\mathcal{O}), \Phi\left(\left(n(x) a_{y}\right)_{\infty} \cdot \gamma_{p} \cdot \prod_{v \neq p, \infty} 1_{v}\right)=\Phi\left(\left(\gamma_{p}^{-1} n(x) a_{y}\right)_{\infty} \prod_{v<\infty} 1_{v}\right)=$ $F\left(\gamma_{p}^{-1} n(x) a_{y}\right)$. Here, we have used the left $\mathcal{G}(\mathbb{Q})$-invariance and right $K_{v^{-}}$ invariance of $\Phi$.

Next, we substitute the Fourier expansion of $F$ and perform an appropriate change of variable for $\beta$ to get the proposition. Note that, for the first set of left cosets, we have to use that $\mathcal{O} / p \mathcal{O} \ni x \mapsto \exp (2 \pi i \operatorname{Re}(\beta x / p))$ is a nontrivial character of $(\mathbb{Z} / p \mathbb{Z})^{4}$ if and only if $p$ does not divide $\beta$. 
For this proposition we remark that the formulas above do not depend on the choices of representatives of $C_{p}$, since $F$ is left- and right-invariant with respect to $\left.\left\{\begin{array}{cc}u_{1} & \\ & u_{2}\end{array}\right] \mid u_{1}, u_{2} \in \mathcal{O}^{\times}\right\}$. Let the Fourier decomposition of $\left(K_{p} h K_{p} \cdot F\right)$ be given by

$$
\left(K_{p} h K_{p} \cdot F\right)\left(n(x) a_{y}\right)=\sum_{\beta \in S \backslash\{0\}}\left(K_{p} h K_{p} \cdot F\right)_{\beta} y^{2} K_{\sqrt{-1} r}(2 \pi|\beta| y) e^{2 \pi \sqrt{-1} \operatorname{Re}(\beta x)} .
$$

The next proposition provides a formula for $\left(K_{p} h K_{p} \cdot F\right)_{\beta}$ in terms of the Fourier coefficients $A(\beta)$ of $F$.

Proposition 5.8.

(1) Let $p=2$, and let $h=\left[\begin{array}{cc}\varpi_{2} & 0 \\ 0 & 1\end{array}\right]$. We obtain

$$
\left(K_{2} h K_{2} \cdot F\right)_{\beta}=2\left(A\left(\beta \varpi_{2}^{-1}\right)+A\left(\beta \varpi_{2}\right)\right) .
$$

(2) Let $p$ be an odd prime, and let $\beta \in S \backslash\{0\}$.

(a) When $h=\left[\begin{array}{lll}p & & \\ & & \\ & p & \\ & & 1\end{array}\right]$,

$$
\left(K_{p} h K_{p} \cdot F\right)_{\beta}=p\left(\sum_{\alpha \in C_{p}} A\left(\beta \bar{\alpha}^{-1}\right)+\sum_{\alpha \in C_{p}} A(\bar{\alpha} \beta)\right) .
$$

(b) When $h=\left[\begin{array}{llll}p & & \\ & 1 & \\ & & 1 & \\ & & 1\end{array}\right]$,

$$
\left(K_{p} h K_{p} \cdot F\right)_{\beta}=p\left(\sum_{\alpha \in C_{p}} A\left(\alpha^{-1} \beta\right)+\sum_{\alpha \in C_{p}} A(\beta \alpha)\right) .
$$

(c) When $h=\left[\begin{array}{lll}p & & \\ & p & \\ & 1 & \\ & & 1\end{array}\right]$,

$$
\begin{aligned}
\left(K_{p} h K_{p} \cdot F\right)_{\beta}= & p^{2}\left(A\left(p^{-1} \beta\right)+A(p \beta)\right) \\
& +p \sum_{\left(\alpha_{1}, \alpha_{2}\right) \in C_{p} \times C_{p}} A\left(\alpha_{1}^{-1} \beta \alpha_{2}\right) .
\end{aligned}
$$


For this proposition we note that the automorphy of $F$ with respect

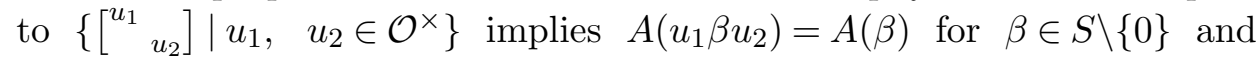
$u_{1}, u_{2} \in \mathcal{O}^{\times}$. From this we see that the formulas in (2b) and (2c) do not depend on the choices of representatives for $C_{p}$. For (2a) we furthermore see that, given any complete set $\left\{\alpha_{i} \mid 0 \leqslant i \leqslant p\right\}$ of representatives for $C_{p}$, $\left\{\bar{\alpha}_{i} \mid 0 \leqslant i \leqslant p\right\}$ also forms such a set. As a result, we see that the formula in (2a) is also not dependent on the choices of representatives for $C_{p}$.

\subsection{Hecke equivariance for $p=2$}

Let $f \in S\left(\Gamma_{0}(2) ;-\left(\frac{1}{4}+r^{2} / 4\right)\right.$ ) be a new form (for the definition see [12, §8.5]) with Hecke eigenvalue $\lambda_{p}$ for $p=2$. By the Hecke eigenvalue $\lambda_{2}$ we mean the eigenvalue of $f$ for the $U(2)$ operator defined by the action of the double coset $\Gamma_{0}(2)\left[{ }_{1}^{1} 2\right] \Gamma_{0}(2)$. Let us also assume that $f$ is an eigenfunction of the Atkin-Lehner involution with eigenvalue $\epsilon$. It can be checked that $\lambda_{2}$ and $\epsilon$ are related by

$$
\lambda_{2}=-\epsilon
$$

Using the single coset decomposition

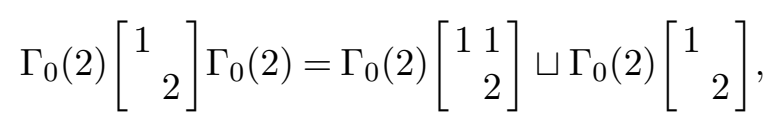

we get

$$
f\left(\frac{z+1}{2}\right)+f\left(\frac{z}{2}\right)=\lambda_{2} f(z) .
$$

In terms of Fourier coefficients of $f$, using (5.5), we get

$$
c(2 m)=\frac{\lambda_{2}}{2} c(m)=-\frac{\epsilon}{2} c(m), \quad \text { for all } m \in \mathbb{Z} .
$$

Proposition 5.9. Let $f \in S\left(\Gamma_{0}(2) ;-\left(\frac{1}{4}+r^{2} / 4\right)\right)$ be a new form and an eigenfunction of the Atkin-Lehner involution with eigenvalue $\epsilon$. Let $F=F_{f}$ be as defined in Theorem 4.4. Then,

$$
\left(K_{2}\left[\begin{array}{cc}
\varpi_{2} & \\
& 1
\end{array}\right] K_{2}\right) F=-3 \sqrt{2} \epsilon F .
$$

Proof. Let $\beta=\varpi_{2}^{u} d \beta_{0}$ be a decomposition according to Proposition 4.2. Hence, $u \geqslant 0, d$ is odd and $\beta_{0} \in S^{\text {prim }}$. Using (5.5) and (5.6), we see that

$$
A(\beta)=\left(2^{u+1}-1\right)|\beta| \sum_{n \mid d} c\left(\frac{-|\beta|^{2}}{2 n^{2}}\right),
$$




$$
\begin{gathered}
A\left(\beta \varpi_{2}\right)=\left(2^{u+2}-1\right) \frac{-\epsilon}{\sqrt{2}}|\beta| \sum_{n \mid d} c\left(\frac{-|\beta|^{2}}{2 n^{2}}\right), \\
A\left(\beta \varpi_{2}^{-1}\right)=\left(2^{u}-1\right)(-\epsilon \sqrt{2})|\beta| \sum_{n \mid d} c\left(\frac{-|\beta|^{2}}{2 n^{2}}\right) .
\end{gathered}
$$

Note that, if $u=0$, then $A\left(\beta \varpi_{2}^{-1}\right)=0$, and so is the right-hand side of the third equality above. We have

$$
\frac{2^{u+2}-1}{\sqrt{2}}+\left(2^{u}-1\right) \sqrt{2}=\frac{3}{\sqrt{2}}\left(2^{u+1}-1\right) .
$$

Hence, we have

$$
2\left(A\left(\beta \varpi_{2}\right)+A\left(\beta \varpi_{2}^{-1}\right)\right)=-3 \sqrt{2} \epsilon A(\beta) .
$$

The proposition now follows from part (1) of Proposition 5.8.

\subsection{Hecke equivariance for odd primes}

We assume that $f \in S\left(\Gamma_{0}(2) ;-\left(\frac{1}{4}+r^{2} / 4\right)\right)$ is a Hecke eigenform with Hecke eigenvalue $\lambda_{p}$ for every odd prime $p$, but do not assume that $f$ is a new form. In terms of Fourier coefficients of $f$, the Hecke relation is given by

$$
p^{1 / 2} c(p n)+p^{-1 / 2} c(n / p)=\lambda_{p} c(n)
$$

where $c(n / p)$ is assumed to be zero if $p$ does not divide $n$. The following lemma will play a key role in the computation of the Hecke operator.

Lemma 5.10. Let $\beta \in S^{\text {prim }}$. Then,

$$
\#\left\{\alpha \in C_{p}: p \mid \beta \alpha\right\}=\#\left\{\alpha \in C_{p}: p \mid \alpha \beta\right\}= \begin{cases}1 & \text { if }\left.p|| \beta\right|^{2} \\ 0 & \text { if } p X|\beta|^{2}\end{cases}
$$

In addition, $p^{2}$ does not divide $\alpha \beta$ or $\beta \alpha$ for any $\alpha \in C_{p}$.

Proof. Note that, by taking conjugates, it is enough to prove the statement of the lemma for $\left\{\alpha \in C_{p}: p \mid \beta \alpha\right\}$ for all $\beta$. Taking norms, it is clear that $p$ does not divide $\beta \alpha$ if $p$ does not divide $|\beta|^{2}$. Hence, assume that $p$ divides $|\beta|^{2}$. Let $\beta=\beta_{1}+\beta_{2} i+\beta_{3} j+\beta_{4} i j$. The conditions $\left.p|| \beta\right|^{2}$ and $p \times \beta$, imply that there is a pair amongst the set $\left\{\beta_{1}, \beta_{2}, \beta_{3}, \beta_{4}\right\}$ which does not satisfy $x^{2}+y^{2} \equiv 0(\bmod p)$. From the proof it will be clear 
that we can take, without loss of generality, $\beta_{3}^{2}+\beta_{4}^{2} \not \equiv 0(\bmod p)$. Let $\alpha=\alpha_{1}+\alpha_{2} i+\alpha_{3} j+\alpha_{4} i j$. The condition $p \mid \beta \alpha$ is equivalent to the following matrix equation modulo $p$ :

$$
\underbrace{\left[\begin{array}{cccc}
\beta_{1} & -\beta_{2} & -\beta_{3} & -\beta_{4} \\
\beta_{2} & \beta_{1} & -\beta_{4} & \beta_{3} \\
\beta_{3} & \beta_{4} & \beta_{1} & -\beta_{2} \\
\beta_{4} & -\beta_{3} & \beta_{2} & \beta_{1}
\end{array}\right]}_{P_{\beta}}\left[\begin{array}{l}
\alpha_{1} \\
\alpha_{2} \\
\alpha_{3} \\
\alpha_{4}
\end{array}\right]=\left[\begin{array}{l}
0 \\
0 \\
0 \\
0
\end{array}\right] .
$$

The matrix $P_{\beta}$ considered over $\mathbb{Z}_{p}$ has rank 2 . By our assumption $\beta_{3}^{2}+\beta_{4}^{2} \not \equiv$ $0(\bmod p)$, we see that the kernel of $P_{\beta}$ is spanned by the first two rows of $P_{\beta}$. Hence, $\alpha$ is given by

$\alpha_{1}=a \beta_{1}+b \beta_{2}, \quad \alpha_{2}=-a \beta_{2}+b \beta_{1}, \quad \alpha_{3}=-a \beta_{3}-b \beta_{4}, \quad \alpha_{4}=-a \beta_{4}+b \beta_{3}$,

for some $a, b \in \mathbb{Z}_{p}$. This gives us $\alpha_{3}^{2}+\alpha_{4}^{2}=\left(a^{2}+b^{2}\right)\left(\beta_{3}^{2}+\beta_{4}^{2}\right) \neq 0$. This is because, by assumption, $\beta_{3}^{2}+\beta_{4}^{2} \neq 0$, and $|\alpha|^{2}=\left(a^{2}+b^{2}\right)|\beta|^{2}$ and $p^{2}$ does not divide $|\alpha|^{2}$.

In [23, page 69], it is shown that the set $S_{1}=\left\{\alpha \in C_{p}: \alpha_{3}^{2}+\alpha_{4}^{2} \not \equiv\right.$ $0(\bmod p)\}$ is in bijection with the set $S_{2}=\left\{(x, y) \in \mathbb{Z}_{p} \times \mathbb{Z}_{p}: x^{2}+y^{2}+1 \equiv\right.$ $0(\bmod p)\}$. The map from $S_{1}$ to $S_{2}$ is given as follows. For $\alpha \in S_{1}$, we obtain $\left(x_{\alpha}, y_{\alpha}\right)$ as the solution to the matrix equation modulo $p$ given by

$$
\left[\begin{array}{cc}
\alpha_{3} & \alpha_{4} \\
-\alpha_{4} & \alpha_{3}
\end{array}\right]\left[\begin{array}{l}
x_{\alpha} \\
y_{\alpha}
\end{array}\right]=\left[\begin{array}{l}
\alpha_{1} \\
\alpha_{2}
\end{array}\right]
$$

One can check that $(x, y) \in S_{2}$ for the following choice of $x$ and $y$ :

$$
x=\frac{-\beta_{1} \beta_{3}-\beta_{2} \beta_{4}}{\beta_{3}^{2}+\beta_{4}^{2}}, \quad y=\frac{\beta_{2} \beta_{3}-\beta_{4} \beta_{1}}{\beta_{3}^{2}+\beta_{4}^{2}} .
$$

If we take $\alpha \in S_{1}$ to be the preimage of the above $(x, y)$, then we can check that $\alpha \in \operatorname{Ker}\left(P_{\beta}\right)$. On the other hand, if $\alpha \in C_{p}$ belongs to $\operatorname{Ker}\left(P_{\beta}\right)$, then it can also be checked that the corresponding $\left(x_{\alpha}, y_{\alpha}\right)$ are equivalent modulo $p$ to those in (5.10). This completes the proof of (5.9). 
If $p^{2}$ divides $\beta \alpha$ or $\alpha \beta$ for some $\alpha \in C_{p}$, then it is clear that $\beta$ cannot be primitive. This completes the proof of the lemma.

Proposition 5.11. Let $f \in S\left(\Gamma_{0}(2) ;-\left(\frac{1}{4}+r^{2} / 4\right)\right)$ be a Hecke eigenform with Hecke eigenvalue $\lambda_{p}$ for every odd prime $p$. Let $F=F_{f}$ be as defined in Theorem 4.4. For an odd prime $p$ we then have

$$
\left(K_{p}\left[\begin{array}{llll}
p & & & \\
& p & & \\
& & p & \\
& & & 1
\end{array}\right] K_{p}\right) F=\left(K_{p}\left[\begin{array}{cccc}
p & & & \\
& 1 & & \\
& & 1 & \\
& & & 1
\end{array}\right] K_{p}\right) F=p(p+1) \lambda_{p} F .
$$

Proof. Using Proposition 5.8 we can show that, if the Fourier coefficients satisfy $A(\beta)=A(\bar{\beta})$ for all $\beta \in S$ and the second equality in (5.11) holds, then so does the first equality. Since the Fourier coefficients of $F=F_{f}$ satisfy the above condition, we are reduced to showing the second equality in (5.11).

We will compute the action of the Hecke operator on the Fourier coefficients $A(\beta)$ of $F$. Since all the computations only involve the prime $p$, it will be enough to consider the case $\beta=p^{s} \beta_{0}$ with $s \geqslant 0$ and $\beta_{0} \in S^{\text {prim }}$. For such a $\beta$, we have

$$
A(\beta)=p^{s}\left|\beta_{0}\right| \sum_{k=0}^{s} c\left(\frac{-\left|\beta_{0}\right|^{2} p^{2 s-2 k}}{2}\right) .
$$

Note that $\alpha^{-1} \beta_{0}=(1 / p) \bar{\alpha} \beta_{0}$ for $\alpha \in B$ with $\nu(\alpha)=p$. Hence, for such $\alpha$, $\alpha^{-1} \beta_{0} \in S$ if and only if $p$ divides $\bar{\alpha} \beta_{0}$.

Let us first consider the case where $p$ does not divide $\left|\beta_{0}\right|^{2}$. Hence, by Lemma 5.10, we see that $\alpha^{-1} \beta_{0} \notin S$ and $p$ does not divide $\beta_{0} \alpha$ for any $\alpha \in C_{p}$. Hence, for any $\alpha \in C_{p}$, we have

$$
A(\beta \alpha)=\sqrt{p} p^{s}\left|\beta_{0}\right| \sum_{k=0}^{s} c\left(\frac{-\left|\beta_{0}\right|^{2} p^{2 s+1-2 k}}{2}\right)
$$

and

$$
A\left(\alpha^{-1} \beta\right)=\frac{1}{\sqrt{p}} p^{s}\left|\beta_{0}\right| \sum_{k=0}^{s-1} c\left(\frac{-\left|\beta_{0}\right|^{2} p^{2 s-1-2 k}}{2}\right) .
$$


Note that, if $s=0$, both the left- and right-hand sides of the last equation are zero. Now, using (5.8), we get for any $\alpha \in C_{p}$

$$
\begin{aligned}
A\left(\alpha^{-1} \beta\right)+A(\beta \alpha)= & p^{s}\left|\beta_{0}\right|\left(\sum _ { k = 0 } ^ { s - 1 } \left(p^{-1 / 2} c\left(\frac{-\left|\beta_{0}\right|^{2} p^{2 s-1-2 k}}{2}\right)\right.\right. \\
& \left.\left.+p^{1 / 2} c\left(\frac{-\left|\beta_{0}\right|^{2} p^{2 s+1-2 k}}{2}\right)\right)+p^{1 / 2} c\left(\frac{-\left|\beta_{0}\right|^{2} p}{2}\right)\right) \\
= & p^{s}\left|\beta_{0}\right|\left(\sum_{k=0}^{s-1} \lambda_{p} c\left(\frac{-\left|\beta_{0}\right|^{2} p^{2 s-2 k}}{2}\right)+\lambda_{p} c\left(\frac{-\left|\beta_{0}\right|^{2}}{2}\right)\right) \\
= & \lambda_{p} A(\beta) .
\end{aligned}
$$

Now, using the fact that the number of elements in $C_{p}$ is $p+1$ and part (2b) of Proposition 5.8, we get the result.

Next, let us assume that $p$ divides $\left|\beta_{0}\right|^{2}$. By Lemma 5.10, there is a unique $\alpha_{1} \in C_{p}$ such that $p$ divides $\beta_{0} \alpha_{1}$, and a unique $\alpha_{2} \in C_{p}$ such that $\alpha_{2}^{-1} \beta_{0} \in S$. If $\alpha \in C_{p}$ but $\alpha \neq \alpha_{1}$, then the formula for $A(\beta \alpha)$ is the same as in (5.12). For $\alpha=\alpha_{1}$, we have

$$
A\left(\beta \alpha_{1}\right)=\sqrt{p} p^{s}\left|\beta_{0}\right| \sum_{k=0}^{s+1} c\left(\frac{-\left|\beta_{0}\right|^{2} p^{2 s+1-2 k}}{2}\right) .
$$

For $\alpha \in C_{p}$ but $\alpha \neq \alpha_{2}$, the formula for $A\left(\alpha^{-1} \beta\right)$ is the same as in (5.13). For $\alpha=\alpha_{2}$, we have

$$
A\left(\alpha_{2}^{-1} \beta\right)=\frac{1}{\sqrt{p}} p^{s}\left|\beta_{0}\right| \sum_{k=0}^{s} c\left(\frac{-\left|\beta_{0}\right|^{2} p^{2 s-1-2 k}}{2}\right) .
$$

Hence, we get the following:

$$
\begin{aligned}
& \sum_{\alpha \in C_{p}}\left(A\left(\alpha^{-1} \beta\right)+A(\beta \alpha)\right) \\
& =p p^{s}\left|\beta_{0}\right|\left[\sum_{k=0}^{s} p^{1 / 2} c\left(\frac{-\left|\beta_{0}\right|^{2} p^{2 s+1-2 k}}{2}\right)+\sum_{k=0}^{s-1} p^{-1 / 2} c\left(\frac{-\left|\beta_{0}\right|^{2} p^{2 s-1-2 k}}{2}\right)\right] \\
& \quad+p^{s}\left|\beta_{0}\right|\left[\sum_{k=0}^{s+1} p^{1 / 2} c\left(\frac{-\left|\beta_{0}\right|^{2} p^{2 s+1-2 k}}{2}\right)+\sum_{k=0}^{s} p^{-1 / 2} c\left(\frac{-\left|\beta_{0}\right|^{2} p^{2 s-1-2 k}}{2}\right)\right]
\end{aligned}
$$




$$
\begin{aligned}
= & p p^{s}\left|\beta_{0}\right|\left[\sum_{k=0}^{s-1} \lambda_{p} c\left(\frac{-\left|\beta_{0}\right|^{2} p^{2 s-2 k}}{2}\right)+p^{1 / 2} c\left(\frac{-\left|\beta_{0}\right|^{2} p}{2}\right)\right] \\
& +p^{s}\left|\beta_{0}\right|\left[\sum_{k=0}^{s} \lambda_{p} c\left(\frac{-\left|\beta_{0}\right|^{2} p^{2 s-2 k}}{2}\right)+p^{1 / 2} c\left(\frac{-\left|\beta_{0}\right|^{2}}{2 p}\right)\right] \\
= & \lambda_{p} A(\beta)+p p^{s}\left|\beta_{0}\right|\left[\sum_{k=0}^{s-1} \lambda_{p} c\left(\frac{-\left|\beta_{0}\right|^{2} p^{2 s-2 k}}{2}\right)\right. \\
& \left.+p^{1 / 2} c\left(\frac{-\left|\beta_{0}\right|^{2} p}{2}\right)+p^{-1 / 2} c\left(\frac{-\left|\beta_{0}\right|^{2}}{2 p}\right)\right] \\
= & \lambda_{p} A(\beta)+p \lambda_{p} A(\beta)=(p+1) \lambda_{p} A(\beta) .
\end{aligned}
$$

This completes the proof of the proposition.

Proposition 5.12. Let $f \in S\left(\Gamma_{0}(2) ;-\left(\frac{1}{4}+r^{2} / 4\right)\right)$ be a Hecke eigenform with Hecke eigenvalue $\lambda_{p}$ for every odd prime $p$. Let $F=F_{f}$ be as defined in Theorem 4.4. For an odd prime $p$ we then have

$$
\left(K_{p}\left[\begin{array}{llll}
p & & & \\
& p & & \\
& & 1 & \\
& & & 1
\end{array}\right] K_{p}\right) F=\left(p^{2} \lambda_{p}^{2}+p^{3}+p\right) F
$$

Proof. First observe that, using (5.8), one can show that, for all $n$,

$$
p c\left(n p^{2}\right)=\left(\lambda_{p}^{2}-1\right) c(n)-p^{-1 / 2} \lambda_{p} c(n / p) .
$$

If we assume that $p \mid n$, then we can get another identity given by

$$
p c\left(n p^{2}\right)+p^{-1} c\left(n / p^{2}\right)=\left(\lambda_{p}^{2}-2\right) c(n) .
$$

As in the proof of Proposition 5.11, we can assume that $\beta=p^{s} \beta_{0}$, where $s \geqslant 0$ and $\beta_{0} \in S^{\text {prim }}$. Let us abbreviate $\nu_{p}(\beta)=s$. For such a $\beta$ we have

$$
A(\beta)=p^{s}\left|\beta_{0}\right| \sum_{k=0}^{s} c\left(\frac{-\left|\beta_{0}\right|^{2} p^{2 s-2 k}}{2}\right) .
$$

Hence,

$$
A(p \beta)=p^{s+1}\left|\beta_{0}\right| \sum_{k=0}^{s+1} c\left(\frac{-\left|\beta_{0}\right|^{2} p^{2 s-2 k+2}}{2}\right)
$$


and

$$
A\left(p^{-1} \beta\right)=p^{s-1}\left|\beta_{0}\right| \sum_{k=0}^{s-1} c\left(\frac{-\left|\beta_{0}\right|^{2} p^{2 s-2 k-2}}{2}\right) .
$$

Next, we need to compute $\sum A\left(\alpha_{1}^{-1} \beta \alpha_{2}\right)$ where the sum is over all $\alpha_{1}, \alpha_{2}$ in $C_{p}$. We consider three cases depending on whether $p \nmid\left|\beta_{0}\right|^{2}$, or $\left.p|| \beta_{0}\right|^{2}$ but $p^{2} \backslash\left|\beta_{0}\right|^{2}$ or $\left.p^{2}|| \beta_{0}\right|^{2}$.

Case 1: Let us assume that $p X\left|\beta_{0}\right|^{2}$. Applying Lemma 5.10 to $\beta_{0}$, we see that $\beta_{0} \alpha_{2} \in S^{\text {prim }}$ for all $\alpha_{2} \in C_{p}$. Again applying Lemma 5.10 to $\beta_{0} \alpha_{2}$ for a fixed $\alpha_{2}$, we see there is a unique $\alpha_{1,2} \in C_{p}$ such that $\nu_{p}\left(\alpha_{1,2}^{-1} \beta \alpha_{2}\right)=s$, and, for all $\alpha_{1} \neq \alpha_{1,2}$, we have $\nu_{p}\left(\alpha_{1}^{-1} \beta \alpha_{2}\right)=s-1$. Hence,

$$
\begin{aligned}
\sum_{\alpha_{1}, \alpha_{2} \in C_{p}} A\left(\alpha_{1}^{-1} \beta \alpha_{2}\right) & =\sum_{\alpha_{2} \in C_{p}}\left(A\left(\alpha_{1,2}^{-1} \beta \alpha_{2}\right)+\sum_{\substack{\alpha_{1} \in C_{p} \\
\alpha_{1} \neq \alpha_{1,2}}} A\left(\alpha_{1}^{-1} \beta \alpha_{2}\right)\right) \\
& =(p+1) A(\beta)+(p+1) p p^{s}\left|\beta_{0}\right| \sum_{k=0}^{s-1} c\left(\frac{-\left|\beta_{0}\right|^{2} p^{2 s-2 k}}{2}\right) .
\end{aligned}
$$

Putting this all together, we see that $p^{2}\left(A(p \beta)+A\left(p^{-1} \beta\right)\right)+$ $p \sum_{\alpha_{1}, \alpha_{2}} A\left(\alpha_{1}^{-1} \beta \alpha_{2}\right)$ is equal to

$$
\begin{aligned}
p^{2}( & \left.p^{s+1}\left|\beta_{0}\right| \sum_{k=0}^{s+1} c\left(\frac{-\left|\beta_{0}\right|^{2} p^{2 s-2 k+2}}{2}\right)+p^{s-1}\left|\beta_{0}\right| \sum_{k=0}^{s-1} c\left(\frac{-\left|\beta_{0}\right|^{2} p^{2 s-2 k-2}}{2}\right)\right) \\
& +p(p+1) A(\beta)+(p+1) p^{2} p^{s}\left|\beta_{0}\right| \sum_{k=0}^{s-1} c\left(\frac{-\left|\beta_{0}\right|^{2} p^{2 s-2 k}}{2}\right) \\
= & p^{2} p^{s}\left|\beta_{0}\right|\left(\left(\lambda_{p}^{2}-2\right) \sum_{k=0}^{s-1} c\left(\frac{-\left|\beta_{0}\right|^{2} p^{2 s-2 k}}{2}\right)+p c\left(\frac{-\left|\beta_{0}\right|^{2} p^{2}}{2}\right)+p c\left(\frac{-\left|\beta_{0}\right|^{2}}{2}\right)\right) \\
& +p(p+1) A(\beta)+(p+1) p^{2} p^{s}\left|\beta_{0}\right| \sum_{k=0}^{s-1} c\left(\frac{-\left|\beta_{0}\right|^{2} p^{2 s-2 k}}{2}\right) \\
= & p^{2} p^{s}\left|\beta_{0}\right|\left(\left(\lambda_{p}^{2}-2\right) \sum_{k=0}^{s} c\left(\frac{-\left|\beta_{0}\right|^{2} p^{2 s-2 k}}{2}\right)\right. \\
& -\left(\lambda_{p}^{2}-2\right) c\left(\frac{-\left|\beta_{0}\right|^{2}}{2}\right)+\left(\lambda_{p}^{2}-1\right) c\left(\frac{-\left|\beta_{0}\right|^{2}}{2}\right)
\end{aligned}
$$




$$
\begin{aligned}
& \left.+p c\left(\frac{-\left|\beta_{0}\right|^{2}}{2}\right)\right)+p(p+1) A(\beta)+(p+1) p^{2} A(\beta) \\
& -(p+1) p^{2} p^{s}\left|\beta_{0}\right| c\left(\frac{-\left|\beta_{0}\right|^{2}}{2}\right) \\
= & \left(p^{2}\left(\lambda_{p}^{2}-2\right)+p(p+1)+p^{2}(p+1)\right) A(\beta) \\
= & \left(p^{2} \lambda_{p}^{2}+p^{3}+p\right) A(\beta) .
\end{aligned}
$$

Here, we have used both (5.15) and (5.16).

Case 2: Let $\left.p|| \beta_{0}\right|^{2}$ but $p^{2} \chi\left|\beta_{0}\right|^{2}$. Applying Lemma 5.10 to $\beta_{0}$, we see that there is a unique $\hat{\alpha}_{2} \in C_{p}$ such that $p \mid \beta_{0} \hat{\alpha}_{2}$. For $\alpha_{2} \neq \hat{\alpha}_{2}$, we have $\beta_{0} \alpha_{2} \in$ $S^{\text {prim }}$. Let $\beta_{0} \hat{\alpha}_{2}=p \beta_{0}^{\prime}$. Then $\beta_{0}^{\prime} \in S^{\text {prim }}$ and $p \nmid\left|\beta_{0}^{\prime}\right|^{2}$ (since we have assumed that $\left.p^{2} \backslash\left|\beta_{0}\right|^{2}\right)$. Hence, by Lemma 5.10, we see that, for all $\alpha_{1} \in C_{p}$, we have $\alpha_{1}^{-1} \beta_{0} \hat{\alpha}_{2}=\bar{\alpha}_{1} \beta_{0}^{\prime} \in S^{\text {prim }}$. This implies that $\nu_{p}\left(\alpha_{1}^{-1} \beta \hat{\alpha}_{2}\right)=s$ for all $\alpha_{1} \in C_{p}$. If $\alpha_{2} \neq \hat{\alpha}_{2}$, then Lemma 5.10 implies that there is a unique $\alpha_{1,2} \in C_{p}$ such that $\nu_{p}\left(\alpha_{1,2}^{-1} \beta \alpha_{2}\right)=s$. For all $\alpha_{1} \neq \alpha_{1,2}$, we have $\nu_{p}\left(\alpha_{1}^{-1} \beta \alpha_{2}\right)=s-1$. This gives us

$$
\begin{aligned}
& \sum_{\alpha_{1}, \alpha_{2} \in C_{p}} A\left(\alpha_{1}^{-1} \beta \alpha_{2}\right) \\
& =\sum_{\alpha_{1} \in C_{p}} A\left(\alpha_{1}^{-1} \beta \hat{\alpha}_{2}\right)+\sum_{\substack{\alpha_{2} \in C_{p} \\
\alpha_{2} \neq \hat{\alpha}_{2}}}\left(A\left(\alpha_{1,2}^{-1} \beta \alpha_{2}\right)+\sum_{\substack{\alpha_{1} \in C_{p} \\
\alpha_{1} \neq \alpha_{1,2}}} A\left(\alpha_{1}^{-1} \beta \alpha_{2}\right)\right) \\
& =(p+1) A(\beta)+p A(\beta)+p^{2} p^{s}\left|\beta_{0}\right| \sum_{k=0}^{s-1} c\left(\frac{-\left|\beta_{0}\right|^{2} p^{2 s-2 k}}{2}\right) .
\end{aligned}
$$

Putting this all together, we see that $p^{2}\left(A(p \beta)+A\left(p^{-1} \beta\right)\right)+$ $p \sum_{\alpha_{1}, \alpha_{2}} A\left(\alpha_{1}^{-1} \beta \alpha_{2}\right)$ is equal to

$$
\begin{aligned}
& p^{2} p^{s}\left|\beta_{0}\right|\left(\left(\lambda_{p}^{2}-2\right) \sum_{k=0}^{s-1} c\left(\frac{-\left|\beta_{0}\right|^{2} p^{2 s-2 k}}{2}\right)+p c\left(\frac{-\left|\beta_{0}\right|^{2} p^{2}}{2}\right)+p c\left(\frac{-\left|\beta_{0}\right|^{2}}{2}\right)\right) \\
& \quad+p(p+1) A(\beta)+p^{2} A(\beta)+p^{3} p^{s}\left|\beta_{0}\right| \sum_{k=0}^{s-1} c\left(\frac{-\left|\beta_{0}\right|^{2} p^{2 s-2 k}}{2}\right) \\
& =p^{2}\left(\lambda_{p}^{2}-2\right) A(\beta)+p^{2} p^{s}\left|\beta_{0}\right|\left(-\left(\lambda_{p}^{2}-2\right) c\left(\frac{-\left|\beta_{0}\right|^{2}}{2}\right)\right. \\
& \left.\quad+\left(\lambda_{p}^{2}-2\right) c\left(\frac{-\left|\beta_{0}\right|^{2}}{2}\right)+p c\left(\frac{-\left|\beta_{0}\right|^{2}}{2}\right)\right)
\end{aligned}
$$




$$
\begin{aligned}
& +p(p+1) A(\beta)+p^{2} A(\beta)+p^{3} A(\beta)-p^{3} p^{s}\left|\beta_{0}\right| c\left(\frac{-\left|\beta_{0}\right|^{2}}{2}\right) \\
= & \left(p^{2}\left(\lambda_{p}^{2}-2\right)+p(p+1)+p^{2}+p^{3}\right) A(\beta) \\
= & \left(p^{2} \lambda_{p}^{2}+p^{3}+p\right) A(\beta) .
\end{aligned}
$$

Here, we have used (5.16) and $\left.p|| \beta_{0}\right|^{2}$.

Case 3: Let $\left.p^{2}|| \beta_{0}\right|^{2}$. As in Case 2 above, Lemma 5.10 applied to $\beta_{0}$ implies that there is a unique $\hat{\alpha}_{2} \in C_{p}$ such that $p \mid \beta_{0} \hat{\alpha}_{2}$. For $\alpha_{2} \neq \hat{\alpha}_{2}$, we have $\beta_{0} \alpha_{2} \in S^{\text {prim }}$. Let $\beta_{0} \hat{\alpha}_{2}=p \beta_{0}^{\prime}$. Then $\beta_{0}^{\prime} \in S^{\text {prim }}$ and $\left.p|| \beta_{0}^{\prime}\right|^{2}$ (since we have assumed $\left.p^{2}|| \beta_{0}\right|^{2}$ ). Hence, by Lemma 5.10, there is a unique $\hat{\alpha}_{1,2} \in C_{p}$ such that $\nu_{p}\left(\hat{\alpha}_{1,2}^{-1} \beta \hat{\alpha}_{2}\right)=s+1$, and for all $\alpha_{1} \neq \hat{\alpha}_{1,2}$, we have $\nu_{p}\left(\alpha_{1}^{-1} \beta \hat{\alpha}_{2}\right)=s$. If $\alpha_{2} \neq \hat{\alpha}_{2}$, then Lemma 5.10 implies that there is a unique $\alpha_{1,2} \in C_{p}$ such that $\nu_{p}\left(\alpha_{1,2}^{-1} \beta \alpha_{2}\right)=s$. For all $\alpha_{1} \neq \alpha_{1,2}$, we have $\nu_{p}\left(\alpha_{1}^{-1} \beta \alpha_{2}\right)=s-1$. This gives us that $\sum A\left(\alpha_{1}^{-1} \beta \alpha_{2}\right)$ is equal to

$$
\begin{aligned}
A\left(\hat{\alpha}_{1,2}^{-1} \beta \hat{\alpha}_{2}\right)+\sum_{\alpha_{1} \neq \hat{\alpha}_{1,2}} A\left(\alpha_{1}^{-1} \beta \hat{\alpha}_{2}\right) \\
+\sum_{\alpha_{2} \neq \hat{\alpha}_{2}}\left(A\left(\alpha_{1,2} \beta \alpha_{2}\right)+\sum_{\alpha_{1} \neq \alpha_{1,2}} A\left(\alpha_{1}^{-1} \beta \alpha_{2}\right)\right) \\
=p^{s}\left|\beta_{0}\right| \sum_{k=0}^{s+1} c\left(\frac{-\left|\beta_{0}\right|^{2} p^{2 s-2 k}}{2}\right)+p A(\beta) \\
\quad+p A(\beta)+p^{2} p^{s}\left|\beta_{0}\right| \sum_{k=0}^{s-1} c\left(\frac{-\left|\beta_{0}\right|^{2} p^{2 s-2 k}}{2}\right) \\
=A(\beta)+p^{s}\left|\beta_{0}\right| c\left(\frac{-\left|\beta_{0}\right|^{2}}{2 p^{2}}\right)+p A(\beta) \\
\quad+p A(\beta)+p^{2} A(\beta)-p^{2} p^{s}\left|\beta_{0}\right| c\left(\frac{-\left|\beta_{0}\right|^{2}}{2}\right) .
\end{aligned}
$$

Putting this all together, we see that $p^{2}\left(A(p \beta)+A\left(p^{-1} \beta\right)\right)+$ $p \sum_{\alpha_{1}, \alpha_{2}} A\left(\alpha_{1}^{-1} \beta \alpha_{2}\right)$ is equal to

$$
\begin{aligned}
& p^{2}\left(\lambda_{p}^{2}-2\right) A(\beta)+p^{2} p^{s}\left|\beta_{0}\right|\left(-\left(\lambda_{p}^{2}-2\right) c\left(\frac{-\left|\beta_{0}\right|^{2}}{2}\right)\right. \\
& \left.\quad+p c\left(\frac{-\left|\beta_{0}\right|^{2} p^{2}}{2}\right)+p c\left(\frac{-\left|\beta_{0}\right|^{2}}{2}\right)\right)
\end{aligned}
$$




$$
\begin{aligned}
& +p\left(1+2 p+p^{2}\right) A(\beta)+p^{2} p^{s}\left|\beta_{0}\right|\left(p^{-1} c\left(\frac{-\left|\beta_{0}\right|^{2}}{2 p^{2}}\right)-p c\left(\frac{-\left|\beta_{0}\right|^{2}}{2}\right)\right) \\
= & \left(p^{2}\left(\lambda_{p}^{2}-2\right)+p\left(1+2 p+p^{2}\right)\right) A(\beta) \\
= & \left(p^{2} \lambda_{p}^{2}+p^{3}+p\right) A(\beta) .
\end{aligned}
$$

Here, we have used (5.16) and $\left.p^{2}|| \beta_{0}\right|^{2}$. This completes the proof of the proposition.

\section{§. The automorphic representation corresponding to the lifting}

In this section, we will use the Hecke equivariance from the previous section to determine the local components of the automorphic representation corresponding to the lifting. This will lead us to the conclusion that we have obtained a CAP representation and have found a counterexample to the Ramanujan conjecture.

\subsection{The local components of the automorphic representation}

Let $f \in S\left(\Gamma_{0}(2) ;-\left(\frac{1}{4}+r^{2} / 4\right)\right)$ be a Hecke eigenform with Hecke eigenvalue $\lambda_{p}$ for every odd prime $p$. Let $F=F_{f}$ be as defined in Theorem 4.4. Let $\Phi_{F}: \mathcal{G}(\mathbb{A}) \rightarrow \mathbb{C}$ be defined by

$$
\Phi_{F}\left(\gamma g_{\infty} u_{f}\right)=F\left(g_{\infty}\right) \quad \forall\left(\gamma, g_{\infty}, u_{f}\right) \in \mathcal{G}(\mathbb{Q}) \times \mathcal{G}(\mathbb{R}) \times U
$$

See Section 5.1 for details. Let $\pi_{F}$ be the irreducible cuspidal automorphic representation of $\mathcal{G}(\mathbb{A})$ generated by the right translates of $\Phi_{F}$. Note that the irreducibility follows from the strong multiplicity-one result for $\mathcal{G}(\mathbb{A})$ (see [2], [3]). The representation $\pi_{F}$ is cuspidal since $F$ is a cusp form. Let $\pi_{F}=\bigotimes_{p}^{\prime} \pi_{p}$, where $\pi_{p}$ is an irreducible admissible representation of $\mathcal{G}_{p}:=$ $\mathcal{G}\left(\mathbb{Q}_{p}\right)$ for $p<\infty$, and $\pi_{\infty}$ is an irreducible admissible representation of $\mathcal{G}(\mathbb{R})$. Recall that $U=\prod_{p<\infty} K_{p}$, where $K_{p}$ is the maximal compact subgroup of $\mathcal{G}\left(\mathbb{Q}_{p}\right)$ (cf. Section 2.3). Hence, for $p<\infty$, the representation $\pi_{p}$ is a spherical representation and can be realized as a subrepresentation of an unramified principal series representation, that is a representation induced from an unramified character of the Borel subgroup. The representation $\pi_{p}$ is completely determined by the action of the Hecke algebra $\mathcal{H}\left(\mathcal{G}_{p}, K_{p}\right)$ on the spherical vector in $\pi_{p}$, which, in turn, is completely determined by the Hecke eigenvalues of $F$ obtained in the previous section. See [4] for details. For $p=2$ we need to assume that $f$ is a new form for the determination of the Hecke eigenvalue of $F_{f}$ (cf. Section 5.3). 
Description of $\pi_{p}$ for $p$ odd

If $p$ is an odd prime, then we have $\mathcal{G}_{p}=\mathrm{GL}_{4}\left(\mathbb{Q}_{p}\right)$ and $K_{p}=\mathrm{GL}_{4}\left(\mathbb{Z}_{p}\right)$. Given 4 unramified characters $\chi_{1}, \chi_{2}, \chi_{3}, \chi_{4}$ of $\mathbb{Q}_{p}^{\times}$, we obtain a character $\chi$ of the Borel subgroup $P$ of upper triangular matrices in $\mathcal{G}$, by

$$
\chi\left(\left[\begin{array}{cccc}
a_{1} & * & * & * \\
& a_{2} & * & * \\
& & a_{3} & * \\
& & & a_{4}
\end{array}\right]\right)=\chi_{1}\left(a_{1}\right) \chi_{2}\left(a_{2}\right) \chi_{3}\left(a_{3}\right) \chi_{4}\left(a_{4}\right) .
$$

The modulus character $\delta_{P}$ is given by

$$
\delta_{P}\left(\left[\begin{array}{cccc}
a_{1} & * & * & * \\
& a_{2} & * & * \\
& & a_{3} & * \\
& & & a_{4}
\end{array}\right]\right)=\left|a_{1}^{3} a_{2} a_{3}^{-1} a_{4}^{-3}\right|_{p}
$$

where $|*|_{p}$ denotes the $p$-adic absolute value. The unramified principal representation corresponding to $\chi$ is given by $I(\chi)$, which consists of locally constant functions $f: \mathrm{GL}_{4}\left(\mathbb{Q}_{p}\right) \rightarrow \mathbb{C}$, satisfying

$$
f(b g)=\delta_{P}(b)^{1 / 2} \chi(b) f(g), \quad \text { for all } b \in P, g \in \mathrm{GL}_{4}\left(\mathbb{Z}_{p}\right) .
$$

The action of the Hecke algebra is as follows. If $\phi \in \mathcal{H}\left(\mathrm{GL}_{4}\left(\mathbb{Q}_{p}\right), \mathrm{GL}_{4}\left(\mathbb{Z}_{p}\right)\right)$ and $f \in I(\chi)$, define

$$
(\phi * f)(g)=\int_{\mathrm{GL}_{4}\left(\mathbb{Q}_{p}\right)} \phi(h) f(g h) d h .
$$

Recall that we have normalized the measure $d h$ on $\mathrm{GL}_{4}\left(\mathbb{Q}_{p}\right)$ so that the volume of $\mathrm{GL}_{4}\left(\mathbb{Z}_{p}\right)$ is 1 . Let $f=f_{0}$, the unique vector in $I(\chi)$ that is rightinvariant under $\mathrm{GL}_{4}\left(\mathbb{Z}_{p}\right)$ and $f_{0}(1)=1$, and let $\phi=\phi_{h}$, a characteristic function of $\mathrm{GL}_{4}\left(\mathbb{Z}_{p}\right) h \mathrm{GL}_{4}\left(\mathbb{Z}_{p}\right)=\bigsqcup_{i} h_{i} \mathrm{GL}_{4}\left(\mathbb{Z}_{p}\right)$. It follows from (6.3) that

$$
\left(\phi_{h} * f_{0}\right)(1)=\sum_{i} f_{0}\left(h_{i}\right)=\mu_{h},
$$

where $\mu_{h}$ is determined by the representation $\pi_{p}$. The Hecke algebra $\mathcal{H}\left(\mathrm{GL}_{4}\left(\mathbb{Q}_{p}\right), \mathrm{GL}_{4}\left(\mathbb{Z}_{p}\right)\right)$ is generated by $\left\{\phi_{1}^{ \pm 1}, \phi_{2}, \phi_{3}, \phi_{4}\right\}$, defined in (5.2).

LEMMA 6.1. Let $\mu_{1}, \mu_{2}, \mu_{3}, \mu_{4}$ be the constants obtained by the action of $\phi_{i}, i=1,2,3,4$, on the spherical vector $f_{0}$ in $\pi_{p}$ according to (6.4). Then $\mu_{1}=1, \mu_{2}=\mu_{4}=p(p+1) \lambda_{p}$ and $\mu_{3}=p^{2} \lambda_{p}^{2}+p^{3}+p$. 
Proof. The lemma follows from the fact that the action of the $p$-adic Hecke algebra on the spherical vector in $\pi_{p}$ is exactly the same as that on $F$ (or $\left.\Phi_{F}\right)$. First note that $\phi_{1}$ acts as the identity operator, which implies that $\mu_{1}=1$. The other Hecke eigenvalues follow from Propositions 5.11 and 5.12 .

Recall that Proposition 5.5 gives the double coset decompositions which can be used to determine the action of $\phi$ on $f_{0}$. Let us abbreviate $\alpha_{i}=\chi_{i}(p)$ for $i=1,2,3,4$. Working in the induced model $I(\chi)$ of $\pi_{p}$, we see that

$$
\begin{aligned}
\left(\phi_{1} * f_{0}\right)(1)= & \alpha_{1} \alpha_{2} \alpha_{3} \alpha_{4} \\
\left(\phi_{2} * f_{0}\right)(1)= & p^{3} p^{-3 / 2} \alpha_{1} \alpha_{2} \alpha_{3}+p p^{1 / 2} \alpha_{1} \alpha_{3} \alpha_{4} \\
& +p^{2} p^{-1 / 2} \alpha_{1} \alpha_{2} \alpha_{4}+p^{3 / 2} \alpha_{2} \alpha_{3} \alpha_{4} \\
= & p^{3 / 2} \alpha_{1} \alpha_{2} \alpha_{3} \alpha_{4}\left(\alpha_{1}^{-1}+\alpha_{2}^{-1}+\alpha_{3}^{-1}+\alpha_{4}^{-1}\right), \\
\left(\phi_{4} * f_{0}\right)(1)= & p^{3} p^{-3 / 2} \alpha_{1}+p p^{1 / 2} \alpha_{3}+p^{2} p^{-1 / 2} \alpha_{2}+p^{3 / 2} \alpha_{4} \\
= & p^{3 / 2}\left(\alpha_{1}+\alpha_{2}+\alpha_{3}+\alpha_{4}\right), \\
\left(\phi_{3} * f_{0}\right)(1)= & p^{4} p^{-2} \alpha_{1} \alpha_{2}+p^{2} \alpha_{2} \alpha_{3}+p p \alpha_{2} \alpha_{4} \\
& +p^{3} p^{-1} \alpha_{1} \alpha_{3}+p^{2} \alpha_{1} \alpha_{4}+p^{2} \alpha_{3} \alpha_{4} \\
= & p^{2}\left(\alpha_{1} \alpha_{2}+\alpha_{2} \alpha_{3}+\alpha_{2} \alpha_{4}+\alpha_{1} \alpha_{3}+\alpha_{1} \alpha_{4}+\alpha_{3} \alpha_{4}\right) .
\end{aligned}
$$

Proposition 6.2. Let $f \in S\left(\Gamma_{0}(2) ;-\left(\frac{1}{4}+r^{2} / 4\right)\right)$ be a Hecke eigenform with Hecke eigenvalue $\lambda_{p}$ for every odd prime $p$. Let $F=F_{f}$ be as defined in Theorem 4.4. Let $\pi_{F}=\bigotimes_{p}^{\prime} \pi_{p}$ be the corresponding irreducible cuspidal automorphic representation of $\mathcal{G}(\mathbb{A})$. For an odd prime $p$, the representation $\pi_{p}$ is the unique spherical constituent of the unramified principal series representation $I(\chi)$, where, up to the action of the Weyl group of $\mathrm{GL}_{4}$, the character $\chi$ is given by

(6.6) $\chi_{3}(p)=p^{-1 / 2} \frac{\lambda_{p}+\sqrt{\lambda_{p}^{2}-4}}{2}, \quad \chi_{4}(p)=p^{-1 / 2} \frac{\lambda_{p}-\sqrt{\lambda_{p}^{2}-4}}{2}$.

Proof. The representation $I(\chi)$ corresponding to $\pi_{p}$ is generated by the spherical vector $f_{0}$, and, hence, it is completely determined by the action of the generators of the Hecke algebra on $f_{0}$. The representation $\pi_{p}$ is also 
determined by the Hecke eigenvalues of $F$ (or $\Phi_{F}$ ) under the Hecke algebra $\mathcal{H}\left(\mathcal{G}_{p}, K_{p}\right)$. Substituting the values of $\alpha_{i}=\chi_{i}(p), i=1,2,3,4$, from (6.6) into (6.5) shows that we get the exact same eigenvalues as in Lemma 6.1. This completes the proof of the proposition.

Let us remark here that we can use Lemma 6.1 to directly solve for $\alpha_{i}$ from (6.5). It is a tedious computation but results in the same answer as in the statement of the above proposition.

\section{Description of $\pi_{2}$}

Recall that $B_{2}=B \otimes_{\mathbb{Q}} \mathbb{Q}_{2}$, where $B$ is a definite quaternion algebra over $\mathbb{Q}$ with discriminant 2 , and $\mathcal{O}_{2}$ is the completion of the Hurwitz order $\mathcal{O}$ at 2 . In this case, $\mathcal{G}_{2}=\mathrm{GL}_{2}\left(B_{2}\right)$ and $K_{2}=\mathrm{GL}_{2}\left(\mathcal{O}_{2}\right)$. Given two unramified characters $\chi_{1}, \chi_{2}$ of $B_{2}^{\times}$, we obtain a character $\chi$ of the Borel subgroup of upper triangular matrices on $\mathcal{G}$ by

$$
\chi\left(\left[\begin{array}{ll}
\alpha & * \\
0 & \beta
\end{array}\right]\right)=\chi_{1}(\alpha) \chi_{2}(\beta) .
$$

The modulus character is given by

$$
\delta\left(\left[\begin{array}{ll}
\alpha & * \\
0 & \beta
\end{array}\right]\right)=|\alpha / \beta|_{2}^{2} .
$$

Here, $\|_{2}$ is the 2-adic absolute value of the reduced norm of $B_{2}$. The unramified principal series representation corresponding to $\chi$ is given by $I(\chi)$, which consists of locally constant functions $f: \mathcal{G}_{2} \rightarrow \mathbb{C}$, satisfying

$$
f(b g)=\delta(b)^{1 / 2} \chi(b) f(g), \quad \text { for all } b \in \text { Borel subgroup, } g \in \mathcal{G}_{2} .
$$

The action of the Hecke algebra is as follows. If $\phi \in \mathcal{H}\left(\mathcal{G}_{2}, K_{2}\right)$ and $f \in I(\chi)$, define

$$
(\phi * f)(g)=\int_{\mathcal{G}_{2}} \phi(h) f(g h) d h .
$$

Recall that we have normalized the measure $d h$ on $\mathcal{G}_{2}$ so that the volume of $K_{2}$ is 1 . Let $f=f_{0}$, the unique vector in $I(\chi)$ that is right-invariant under $K_{2}$ and $f_{0}(1)=1$, and let $\phi=\phi_{h}$, a characteristic function of $K_{2} h K_{2}=\bigsqcup_{i} h_{i} K_{2}$. It follows from (6.3) that

$$
\left(\phi_{h} * f_{0}\right)(1)=\sum_{i} f_{0}\left(h_{i}\right)=\mu_{h},
$$


where $\mu_{h}$ is determined by the representation $\pi_{2}$. The Hecke algebra $\mathcal{H}\left(\mathcal{G}_{2}, K_{2}\right)$ is generated by $\left\{\varphi_{1}^{ \pm 1}, \varphi_{2}\right\}$, where $\varphi_{1}, \varphi_{2}$ denote the characteristic functions for

$$
K_{2}\left[\begin{array}{cc}
\varpi_{2} & 0 \\
0 & \varpi_{2}
\end{array}\right] K_{2}, \quad K_{2}\left[\begin{array}{cc}
\varpi_{2} & 0 \\
0 & 1
\end{array}\right] K_{2} .
$$

Here, $\varpi_{2}$ is a uniformizer for $B_{2}$.

LEMMA 6.3. Let $\mu_{1}, \mu_{2}$ be the constants obtained by the action of $\phi_{i}, i=$ 1,2 , on the spherical vector $f_{0}$ in $\pi_{2}$ according to (6.8). Then $\mu_{1}=1$ and $\mu_{2}=-3 \sqrt{2} \epsilon$, where $\epsilon$ is the Atkin-Lehner eigenvalue of $f$.

Proof. The proof is the same as in the case of an odd prime.

Recall that Proposition 5.5 gives the double coset decompositions which can be used to determine the action of $\phi$ on $f_{0}$. Let us abbreviate $\alpha_{i}=\chi_{i}\left(\varpi_{2}\right)$ for $i=1,2$. Working in the induced model $I(\chi)$ of $\pi_{2}$, we see that

$$
\begin{aligned}
& \left(\varphi_{1} * f_{0}\right)(1)=\alpha_{1} \alpha_{2}, \\
& \left(\varphi_{2} * f_{0}\right)(1)=2\left(\alpha_{1}+\alpha_{2}\right) .
\end{aligned}
$$

Proposition 6.4. Let $f \in S\left(\Gamma_{0}(2) ;-\left(\frac{1}{4}+r^{2} / 4\right)\right)$ be a new form with Hecke eigenvalue $\lambda_{p}$ for $p=2$ and Atkin-Lehner eigenvalue $\epsilon$, for which $\lambda_{2}=-\epsilon$ holds (cf. (5.5)). Let $F=F_{f}$ be as defined in Theorem 4.4. Let $\pi_{F}=$ $\bigotimes_{p}^{\prime} \pi_{p}$ be the corresponding irreducible cuspidal automorphic representation of $\mathcal{G}(\mathbb{A})$. The representation $\pi_{2}$ is the unique spherical constituent of the unramified principal series representation $I(\chi)$, where, up to the action of the Weyl group, the character $\chi$ is given by

$$
\chi_{1}\left(\varpi_{2}\right)=-\sqrt{2} \epsilon, \quad \chi_{2}\left(\varpi_{2}\right)=-1 / \sqrt{2} \epsilon .
$$

Proof. The proof is the same as in the case of an odd prime.

\section{Description of $\pi_{\infty}$}

We now determine $\pi_{\infty}$. We note that $F=F_{f} \in \mathcal{M}\left(\mathrm{GL}_{2}(\mathcal{O}) ; r\right)$ implies that the archimedean component $\pi_{\infty}$ of $\pi_{F}$ is spherical. Namely, $\pi_{\infty}$ has a $K_{\infty}$-invariant vector, where we put $K_{\infty}:=K$ with $K$ as in (2.1). In fact, up to constant multiples, such a vector is unique for $\pi_{\infty}$, as we soon see.

We now introduce $M_{\infty}:=\left\{\left(\begin{array}{cc}u_{1} & 0 \\ 0 & u_{2}\end{array}\right) \mid u_{1}, u_{2} \in \mathbb{H}^{1}\right\}$ (see Section 2.1 for $\mathbb{H}^{1}$ ). Let $P_{\infty}$ be the standard proper parabolic subgroup $\mathcal{G}_{\infty}=\mathrm{GL}_{2}(\mathbb{H})$ given by

$$
\left\{\left(\begin{array}{ll}
a & * \\
0 & d
\end{array}\right) \in \mathcal{G}_{\infty}\right\} .
$$


We have $P_{\infty}:=Z^{+} N A M_{\infty}$, where $Z^{+}, N$ and $A$ are as in (2.1). The group $Z^{+} A M_{\infty}$ is nothing but the Levi subgroup of $P_{\infty}$. We now note that the Langlands classification of real reductive groups (cf. [18]) implies that $\pi_{\infty}$ has to be embedded into some principal series representation $I_{P_{\infty}}$ of $\mathcal{G}_{\infty}$ induced from a quasicharacter of $P_{\infty}$. Since $\pi_{\infty}$ is spherical, $I_{P_{\infty}}$ is also spherical. As $\pi_{\infty}$ has the trivial central character, so does $I_{P_{\infty}}$. Combining these with the Frobenius reciprocity for compact Lie groups (cf. [15, Theorem 9.9]), one can verify that the quasicharacter of $P_{\infty}$ inducing $I_{P_{\infty}}$ has to be trivial on $Z^{+} M_{\infty}$, and that $I_{P_{\infty}}$ and $\pi_{\infty}$ have a unique $K_{\infty^{-}}$ invariant vector, up to constant multiples. For $s \in \mathbb{C}$ we introduce the quasicharacter $\chi_{s}$ of $P_{\infty}$ defined by

$$
\chi_{s}\left(\left(\begin{array}{ll}
a & * \\
0 & d
\end{array}\right)\right)=\nu\left(a d^{-1}\right)^{s},
$$

where we recall that $\nu$ denotes the reduced norm of $\mathbb{H}$ (cf. Section 2.1). We note that the quasicharacters of $P_{\infty}$ trivial on $Z^{+} M_{\infty}$ should be of this form. We furthermore introduce the modulus character $\delta_{\infty}$ of $P_{\infty}$. The principal series representation $I_{P_{\infty}}$ is then expressed as

$$
I_{P_{\infty}}=\operatorname{Ind}_{P_{\infty}}^{\mathcal{G}_{\infty}}\left(\delta_{\infty} \chi_{s}\right)
$$

Proposition 6.5. We have an isomorphism

$$
\pi_{\infty} \simeq \operatorname{Ind}_{P_{\infty}}^{\mathcal{G}_{\infty}}\left(\delta_{\infty} \chi_{ \pm \sqrt{-1} r}\right)
$$

as $\left(\mathfrak{g}, K_{\infty}\right)$-modules, where we recall that $\mathfrak{g}$ denotes the Lie algebra of $\mathcal{G}_{\infty}$ (cf. Section2.2).

Proof. Let $v$ be a unique $K_{\infty}$-invariant vector in the representation space of $\operatorname{Ind}_{P_{\infty}}^{\mathcal{G}_{\infty}}\left(\delta_{\infty} \chi_{s}\right)$, which $\pi_{\infty}$ can be embedded into. Then, $v$ can also be regarded as a vector of $\pi_{\infty}$. We remark that $\pi_{\infty}$ can be viewed as a representation of $S L_{2}(\mathbb{H}) \simeq \mathrm{GL}_{2}(\mathbb{H}) / Z^{+}$(cf. Section 2.2) since it has the trivial central character. Consider the infinitesimal action of the Casimir operator $\Omega$ (cf. (2.3)) on $v$. We then have

$$
\Omega \cdot v=\frac{1}{2}\left(\frac{s^{2}}{4}-1\right) v=\frac{1}{2}\left(-\frac{r^{2}}{4}-1\right) v,
$$

which leads to $s= \pm \sqrt{-1} r$. Now recall that we can assume $r \in \mathbb{R}$ (cf. Proposition 2.4). We thus know that the quasi-character $\chi_{s}$ is parametrized by 
a purely imaginary number $\pm \sqrt{-1} r$. By Harish-Chandra $[10, \S 41$, Theorem 1], the spherical principal series representation $\operatorname{Ind}_{P_{\infty}}^{\mathcal{G}_{\infty}}\left(\delta_{\infty} \chi_{ \pm \sqrt{-1} r}\right)$ is an irreducible unitary representation. For this see also [6, Remark (2.1.13)] and note the accidental isomorphism $\operatorname{Spin}(5,1) \simeq \mathrm{SL}_{2}(\mathbb{H})$ as real Lie groups. Consequently we have the isomorphism in the assertion.

\subsection{CAP representations}

Let us first give the definition of CAP representations.

Definition 6.6. Let $G_{1}$ and $G_{2}$ be two reductive algebraic groups over a number field such that $G_{1, v} \simeq G_{2, v}$ for almost all places $v$. Let $P_{2}$ be a parabolic subgroup of $G_{2}$ with Levi decomposition $P_{2}=M_{2} N_{2}$. An irreducible cuspidal automorphic representation $\pi=\bigotimes_{v}^{\prime} \pi_{v}$ of $G_{1}(\mathbb{A})$ is called cuspidal associated to parabolic (CAP) $P_{2}$, if there exists an irreducible cuspidal automorphic representation $\sigma$ of $M_{2}$ such that $\pi_{v} \simeq \pi_{v}^{\prime}$ for almost all places $v$, where $\pi^{\prime}=\bigotimes_{v}^{\prime} \pi_{v}^{\prime}$ is an irreducible constituent of $\operatorname{Ind}_{P_{2}(\mathbb{A})}^{G_{2}(\mathbb{A})}(\sigma)$.

See [8] and [22] for details on CAP representations defined for two groups instead of just one. Take $G_{1}=\mathcal{G}=\mathrm{GL}_{2}(B)$ and $G_{2}=\mathrm{GL}_{4}$. Here, $B$ is a definite quaternion algebra with discriminant 2 . Since these groups are inner forms of each other, we have $G_{1, p} \simeq G_{2, p}$ for all odd primes $p$. Let $P_{2}$ be the standard parabolic of $\mathrm{GL}_{4}$ with Levi subgroup $M_{2}=\mathrm{GL}_{2} \times \mathrm{GL}_{2}$. Let $f \in S\left(\Gamma_{0}(2) ;-\left(\frac{1}{4}+r^{2} / 4\right)\right)$ be a Hecke eigenform with Hecke eigenvalue $\lambda_{p}$ for every odd prime $p$ and Atkin-Lehner eigenvalue $\epsilon$. Let $\sigma=\bigotimes_{p}^{\prime} \sigma_{p}$ be the irreducible cuspidal automorphic representation of $\mathrm{GL}_{2}$ corresponding to $f$. For an odd prime $p$, the representation $\sigma_{p}$ is the unramified principal series representation $I(\eta)$, where $\eta$ is given by

$$
\eta\left(\left[\begin{array}{rr}
a & b \\
& d
\end{array}\right]\right)=\eta_{0}(a) \eta_{0}^{-1}(d) .
$$

Here, $\eta_{0}$ is an unramified character of $\mathbb{Q}_{p}^{\times}$such that $\eta_{0}(p)+\eta_{0}^{-1}(p)=\lambda_{p}$. For $p=2$ assume that $f$ is a new form. Then, the representation $\sigma_{2}$ is the twist of the Steinberg representation of $\mathrm{GL}_{2}\left(\mathbb{Q}_{2}\right)$ by an unramified character $\eta^{\prime}$, with $\eta^{\prime}(2)=-\epsilon$. The representation $\sigma$ gives a representation $|\operatorname{det}|_{\mathbb{A}}^{-1 / 2} \sigma \times$ $|\operatorname{det}|_{\mathbb{A}}^{1 / 2} \sigma$ of $M_{2}$, where ||$_{\mathbb{A}}$ denotes the idele norm of $\mathbb{A}^{\times}$. We have the following theorem.

THEOREM 6.7. Let $f \in S\left(\Gamma_{0}(2) ;-\left(\frac{1}{4}+r^{2} / 4\right)\right)$ be a Hecke eigenform with Hecke eigenvalue $\lambda_{p}$ for every odd prime $p$ and Atkin-Lehner eigenvalue $\epsilon$. 
Let $\sigma=\bigotimes_{p}^{\prime} \sigma_{p}$ be the irreducible cuspidal automorphic representation of $\mathrm{GL}_{2}$ corresponding to $f$. Let $F=F_{f}$ be as defined in Theorem 4.4. Let $\pi_{F}=\bigotimes_{p}^{\prime} \pi_{p}$ be the corresponding irreducible cuspidal automorphic representation of $\mathcal{G}(\mathbb{A})$. Then $\pi_{F}$ is CAP to an irreducible constituent of $\operatorname{Ind}_{P_{2}(\mathbb{A})}^{G_{2}(\mathbb{A})}\left(|\operatorname{det}|_{\mathbb{A}}^{-1 / 2} \sigma \times|\operatorname{det}|_{\mathbb{A}}^{1 / 2} \sigma\right)$.

Proof. The theorem follows from the observation that, for an odd prime $p$, we have the isomorphism $\operatorname{Ind}_{P_{2}\left(\mathbb{Q}_{p}\right)}^{G_{2}}\left(|\operatorname{det}|_{p}^{-1 / 2} \sigma_{p} \times|\operatorname{det}|_{p}^{1 / 2} \sigma_{p}\right) \simeq$ $I\left(\chi_{p}\right)$. Here, $I\left(\chi_{p}\right)$ is the representation described in Proposition 6.2. A concrete map is given as follows. For $s \in \operatorname{Ind}_{P_{2}\left(\mathbb{Q}_{p}\right)}^{G_{2}\left(\mathbb{Q}_{p}\right)}\left(|\operatorname{det}|_{p}^{-1 / 2} \sigma_{p} \times|\operatorname{det}|_{p}^{1 / 2} \mid \sigma_{p}\right)$ define the function $g \mapsto(s(g))\left(I_{2}, I_{2}\right)$. Note that $\delta_{P_{2}}\left(\operatorname{diag}\left(a_{1}, a_{2}, a_{3}, a_{4}\right)\right)=$ $\left|a_{1} a_{2} a_{3}^{-1} a_{4}^{-1}\right|_{p}^{2}$.

We can furthermore show that our cuspidal representations $\pi_{F}$ provide counterexamples to the Ramanujan conjecture.

Theorem 6.8. Let $\pi_{F}=\bigotimes_{p}^{\prime} \pi_{p}$ be as in Theorem 6.7. For every odd prime $p$ (respectively $p=\infty$ ), $\pi_{p}$ is nontempered (respectively tempered). If we further assume that $f$ is a new form, $\pi_{p}$ is nontempered for every finite prime $p$ and tempered for $p=\infty$.

Proof. The temperedness of $\pi_{\infty}$ is due to Proposition 6.5 and [6, Remark 2.1.13]. For an odd prime $p$, the unramified characters $\chi_{i}$ with $1 \leqslant i \leqslant 4$ are not unitary (cf. (6.6)). This means that $\pi_{p}$ is nontempered (cf. [27, (9)]).

Let $p=2$, and suppose that $f$ is a new form. We recall that $f_{0}$ denotes the spherical vector in $\pi_{2}$, and introduce its dual vector $f_{0}^{\prime}$ in the contragredient representation of $\pi_{2}$. With the invariant measure $d g$ of $\mathcal{G}_{p} / Z_{p}$ normalized so that $\int_{K_{2} / Z_{p}} d g=1$, for any $\delta>0$, we consider the following integral of the matrix coefficient:

$$
\int_{\mathcal{G}_{2} / Z_{2}}\left|\left\langle\pi_{2}(g) f_{0}, f_{0}^{\prime}\right\rangle\right|^{2+\delta} d g
$$

over $\mathcal{G}_{2}$ modulo center $Z_{2}$, where $\langle *, *\rangle$ denotes the canonical pairing of $\pi_{2}$ and its contragredient. If $\pi_{2}$ is tempered, this integral should be convergent. Now we note that the set $\left(\bigsqcup_{n \geqslant 0} K_{2}\left(\begin{array}{cc}\varpi_{2}^{n} & 0 \\ 0 & 1\end{array}\right) K_{2}\right) / Z_{2}$ can be regarded as a subdomain of $\mathcal{G}_{2} / Z_{2}$ and that there is a decomposition

$$
K_{2}\left(\begin{array}{cc}
\varpi_{2}^{n} & 0 \\
0 & 1
\end{array}\right) K_{2}=\bigsqcup_{x \in \mathcal{O}_{2} / \varpi_{2}^{n} \mathcal{O}_{2}}\left(\begin{array}{cc}
\varpi_{2}^{n} & 0 \\
0 & 1
\end{array}\right)\left(\begin{array}{cc}
1 & \varpi_{2}^{-n} x \\
0 & 1
\end{array}\right) K_{p} \sqcup\left(\begin{array}{cc}
1 & 0 \\
0 & \varpi_{2}^{n}
\end{array}\right) K_{p} .
$$


It is verified that the Hecke operator defined by $K_{2}\left(\begin{array}{cc}\varpi_{2}^{n} & 0 \\ 0 & 1\end{array}\right) K_{2}$ acts on $f_{0}$ as follows:

$$
\left(K_{2}\left(\begin{array}{cc}
\varpi_{2}^{n} & 0 \\
0 & 1
\end{array}\right) K_{2}\right) \cdot f_{0}=(-\epsilon)^{n}\left(2^{3 n / 2}+2^{n / 2}\right) f_{0} .
$$

We thereby have a divergent integral

$$
\begin{aligned}
& \int\left(\sqcup_{n \geqslant 0} K_{2}\left(\begin{array}{cc}
\varpi_{2}^{n} & 0 \\
0 & 1
\end{array}\right) K_{2}\right) / Z_{p}\left|\left\langle\pi_{2}(g) f_{0}, f_{0}^{\prime}\right\rangle\right|^{2+\delta} d g \\
& =\left(\sum_{n \geqslant 0}\left(2^{3 n / 2}+2^{n / 2}\right)^{2+\delta}\right)\left|\left\langle f_{0}, f_{0}^{\prime}\right\rangle\right|^{2+\delta}=\infty,
\end{aligned}
$$

which leads to a contradiction. We therefore see that $\pi_{2}$ is nontempered. As a result we are done.

REMARK 6.9.

(1) According to Tadić [28], the parabolic induction $I(\chi)$ for $p=2$ (cf. Section 6.1) has two composition factors, one of which is a unique essentially square integrable subquotient. Our nontempered representation $\pi_{2}$ is the remaining nonsquare integrable composition factor. Besides our approach, there seem to be several ways to prove that the nonsquare integrable composition factor is nontempered. In fact, Tadic pointed out that the nontemperedness is proved by using the classification of the nonunitary dual of $\mathrm{GL}(n)$ over a division algebra (cf. [28]) or by Casselman's criterion on the temperedness of an admissible representation.

(2) From Weyl's law (cf. $[12,(11.5)]$ ), we can deduce that there exist nonzero new forms in $S\left(\Gamma_{0}(2) ;-\left(r^{2} / 4+\frac{1}{4}\right)\right)$ for some $r \in \mathbb{R}$. Let $N_{\Gamma}(T)$ be the counting function of an orthogonal basis of the discrete spectrum for a congruence subgroup $\Gamma$, as in $[12, \S 11.1]$. Put $N_{\Gamma_{0}(2)}^{*}(T)$ to be such a counting function for new forms of $\Gamma_{0}(2)$. With the help of Casselman's local theory of old forms and new forms (cf. [5]), we deduce

$$
\begin{aligned}
N_{\Gamma_{0}(2)}^{*}(T) & =\frac{\operatorname{Vol}\left(\mathfrak{h} / \Gamma_{0}(2)\right)-2 \operatorname{Vol}\left(\mathfrak{h} / S L_{2}(\mathbb{Z})\right)}{4 \pi} T^{2}+O(T \log T) \\
& =\frac{\operatorname{Vol}\left(\mathfrak{h} / S L_{2}(\mathbb{Z})\right)}{4 \pi} T^{2}+O(T \log T)
\end{aligned}
$$


from Weyl's law just mentioned. In view of Theorems 4.4 and 6.8 , this leads to the existence of a nonzero cuspidal representation $\pi_{F}$ whose local component $\pi_{p}$ is nontempered at every $p<\infty$. We remark that the formula for $N_{\Gamma_{0}(2)}^{*}(T)$ can be generalized to the case of any prime level.

Acknowledgments. From Masao Tsuzuki we have learned Weyl's law for new Maass cusp forms for $\Gamma_{0}(2)$, which leads to the existence of a nonzero cuspidal representation $\pi_{F}=\bigotimes_{p \leqslant \infty}^{\prime} \pi_{p}$ with nontempered local components $\pi_{p}$ at every $p<\infty$. Marko Tadić kindly made his remark on the proof of the nontemperedness of $\pi_{2}$. These are summarized as Remark 6.9 above. Our deep gratitude is due to them. We would like to thank A. Raghuram for informing us of the paper [9] by Grobner. Our thanks are also due to Harald Grobner for his comment on this paper. We would also like to thank Abhishek Saha for several discussions leading to the proof of the nonvanishing of the lifting. We finally express our profound gratitude to the referee for careful reading and fruitful comments.

\section{REFERENCES}

[1] G. Andrews, R. Askey and R. Roy, Special Functions, Encyclopedia of Mathematics and its Applications 71, Cambridge University Press, Cambridge, 1999.

[2] A. Badulescu, Global Jacquet-Langlands correspondence, multiplicity one and classification of automorphic representations, with an appendix by Neven Grbac, Invent. Math. 172(2) (2008), 383-438.

[3] A. Badulescu and D. Renard, Unitary dual of $\mathrm{GL}(n)$ at archimedean places and global Jacquet-Langlands correspondence, Compos. Math. 146(5) (2010), 1115-1164.

[4] P. Cartier, Representations of $\mathfrak{p}$-adic groups: a survey, Proc. Sympos. Pure Math. 33(1) (1979), 111-155.

[5] W. Casselman, On some results of Atkin and Lehner, Math. Ann. 201 (1973), 301-314.

[6] D. Collingwood, Representations of Rank One Lie Groups, Pitman (Advanced Publishing Program), Boston, 1985.

[7] W. Duke and O. Imamoḡlu, A converse theorem and the Saito Kurokawa lift, Int. Math. Res. Not. IMRN 1996(7) (1996), 347-355.

[8] W. Gan, "The Saito-Kurokawa space of $\mathrm{PGSp}_{4}$ and its transfer to inner forms", Eisenstein series and applications, Progr. Math. 258, 87-123. Birkhäuser, Boston, 2008.

[9] H. Grobner, Automorphic forms, cohomology and CAP representations. The case $\mathrm{GL}_{2}$ over a definite quaternion algebra, J. Ramanujan Math. Soc. 28 (2013), 19-48.

[10] Harish Chandra, Harmonic analysis on real reductive groups III: the Maass-Selberg relations and the Plancherel formula, Ann. of Math. (2) 104 (1976), 117-201.

[11] R. Howe and I.I. Piatetski-Shapiro, A counterexample to the "generalized Ramanujan conjecture" for (quasi-) split groups, Proc. Sympos. Pure Math. 33(1) (1979), $315-322$. 
[12] H. Iwaniec, Spectral Methods of Automorphic Forms, 2nd ed., American Mathematical Society, Revista Matemática Iberoamericana, 2002.

[13] N. Iwahori and H. Matsumoto, On some Bruhat decomposition and the structure of the Hecke rings of p-adic Chevalley groups, Publ. Math. Inst. Hautes Études Sci. 25 (1965), 5-48.

[14] H. Jacquet and J. Shalika, On Euler products and the classification of automorphic forms II, Amer. J. Math. 103 (1981), 777-815.

[15] A. Knapp, Lie Groups Beyond an Introduction, 2nd ed., Progress in Mathematics 140, Birkhäuser, Boston, 2002.

[16] A. Krieg, Modular Forms on Half-Spaces of Quaternions, Lecture Notes in Mathematics 1143, Springer, Berlin, 1985.

[17] N. Kurokawa, Examples of eigenvalues of Hecke operators on Siegel cusp forms of degree two, Invent. Math. 49 (1978), 149-165.

[18] R. Langlands, "On the classification of irreducible representations of real algebraic groups", in Representation theory and Harmonic Analysis on Semisimple Lie Groups, Mathematical Surveys and Monographs 31, American Mathematical Society, 1989, $101-170$.

[19] H. Maass, Automorphe Funktionen von meheren Veränderlichen und Dirchletsche Reihen, Abh. Math. Semin. Univ. Hambg. 16(3-4) (1949), 72-100.

[20] T. Miyake, Modular Forms, Springer, Berlin, 2006.

[21] F. Oliver, D. Lozier, R. Boisvert and C. Clark, NIST Handbook of Mathematical Functions, Cambridge University Press, 2010.

[22] A. Pitale, Lifting from $\widetilde{\mathrm{SL}(2)}$ to GSpin(1,4), Int. Math. Res. Not. IMRN 2005(63) (2005), 3919-3966.

[23] A. Pitale, Lifting from $\widetilde{\operatorname{SL}(2)}$ to GSpin(1,4). Ph.D. thesis, 2006.

[24] V. Platonov and A. Rapinchuk, Algebraic Groups and Number Theory, Pure and Applied Mathematics 139, Academic Press, Boston, 1994.

[25] I. I. Piatetski-Shapiro, On the Saito-Kurokawa lifting, Invent. Math. 71 (1983), 309-338.

[26] I. Satake, Theory of spherical functions on reductive groups over p-adic fields, Publ. Math. Inst. Hautes Etudes Sci. 18 (1963), 5-69.

[27] P. Sarnak, "Notes on the Generalized Ramanujan Conjectures", in Harmonic Analysis, the Trace Formula, and Shimura Varieties, Clay Math. Proc. 4, Amer. Math. Soc., Providence, RI, 2005, 659-685.

[28] M. Tadić, Induced representations of $\mathrm{GL}(n, A)$ for $p$-adic division algebras $A$, J. Reine Angew. Math. 405 (1990), 48-77.

\author{
Masanori Muto \\ Kumamoto Prefectural Toryo High School, 5-10 \\ Komine 4-chome \\ Higashi-ku \\ Kumamoto 862-0933 \\ Japan
}


Hiro-aki Narita

Graduate School of Science and Technology

Kumamoto University

Kurokami

Chuo-ku

Kumamoto 860-8555

Japan

narita@sci.kumamoto-u.ac.jp

Ameya Pitale

Department of Mathematics

University of Oklahoma

Norman

Oklahoma

USA

apitale@ou.edu 